\title{
Patterns of gene recombination shape var gene repertoires in Plasmodium falciparum: comparisons of geographically diverse isolates
}

\author{
Susan M Kraemer*1, Sue A Kyes², Gautam Aggarwal ${ }^{1}$, Amy L Springer1, \\ Siri O Nelson ${ }^{1}$, Zoe Christodoulou ${ }^{2}$, Leia M Smith¹, Wendy Wang1, \\ Emily Levin ${ }^{1}$, Christopher I Newbold ${ }^{2}$, Peter J Myler ${ }^{1,3}$ and \\ Joseph D Smith*1,3
}

Address: ${ }^{1}$ Seattle Biomedical Research Institute, 307 Westlake Avenue North, Suite 500, Seattle, WA 98109-5219, USA, ${ }^{2}$ Molecular Parasitology Group, Weatherall Institute of Molecular Medicine, University of Oxford, John Radcliffe Hospital, Oxford OX3 9DS, UK and ${ }^{3}$ Department of Pathobiology, University of Washington, Seattle, WA 98195, USA

Email: Susan M Kraemer* - sue.kraemer@sbri.org; Sue A Kyes - skyes@molbiol.ox.ac.uk; Gautam Aggarwal - g_gautam2000@yahoo.com; Amy L Springer - aspringe@mtholyoke.edu; Siri O Nelson - sirin@u.washington.edu; Zoe Christodoulou - zchristo@hammer.imm.ox.ac.uk; Leia M Smith - lsmith@seagen.com; Wendy Wang - wendywang3@yahoo.com; Emily Levin - emily.levin@sbri.org; Christopher I Newbold - cnewbold@ hammer.imm.ox.ac.uk; Peter J Myler - peter.myler@sbri.org; Joseph D Smith* - joe.smith@sbri.org

* Corresponding authors

Published: 7 February 2007

BMC Genomics 2007, 8:45 doi:10.1 186/147/-2/64-8-45
Received: 7 December 2006

Accepted: 7 February 2007

This article is available from: http://www.biomedcentral.com/I47/-2/64/8/45

(C) 2007 Kraemer et al; licensee BioMed Central Ltd.

This is an Open Access article distributed under the terms of the Creative Commons Attribution License (http://creativecommons.org/licenses/by/2.0), which permits unrestricted use, distribution, and reproduction in any medium, provided the original work is properly cited.

\begin{abstract}
Background: Var genes encode a family of virulence factors known as PfEMPI (Plasmodium falciparum erythrocyte membrane protein I) which are responsible for both antigenic variation and cytoadherence of infected erythrocytes. Although these molecules play a central role in malaria pathogenesis, the mechanisms generating variant antigen diversification are poorly understood. To investigate var gene evolution, we compared the variant antigen repertoires from three geographically diverse parasite isolates: the 3D7 genome reference isolate; the recently sequenced HB3 isolate; and the IT4/25/5 (IT4) parasite isolate which retains the capacity to cytoadhere in vitro and in vivo.
\end{abstract}

Results: These comparisons revealed that only two var genes (varlcsa and var2csa) are conserved in all three isolates and one var gene (Type 3 var) has homologs in IT4 and 3D7. While the remaining 50 plus genes in each isolate are highly divergent most can be classified into the three previously defined major groups (A, B, and $C$ ) on the basis of $5^{\prime}$ flanking sequence and chromosome location. Repertoire-wide sequence comparisons suggest that the conserved homologs are evolving separately from other var genes and that genes in group A have diverged from other groups.

Conclusion: These findings support the existence of a var gene recombination hierarchy that restricts recombination possibilities and has a central role in the functional and immunological adaptation of var genes. 


\section{Background}

Malaria pathogenesis poses a major hindrance to development in many parts of the world with more than 500 million people suffering from the disease and at least one million dying from $P$. falciparum infection each year [1]. Disease severity has been associated with the accumulation of infected erythrocytes (IEs) in microvasculature of vital organs, such as the brain and placenta [2]. A key protein family involved in IE binding is the antigenic variant P. falciparum Erythrocyte Membrane 1 (PfEMP1) [3-5]. Each parasite genome contains about 60 var genes that encode PfEMP1 proteins [6], which are expressed in a mutually exclusive fashion at the IE surface $[7,8]$. Switches in var gene expression allow parasites to evade the host antibody response and sequester at different microvascular sites in the body [9]. Therefore, further definition of the var gene family conservation, the factors regulating variant antigen gene diversification, and the expression of particular var genes during disease will provide critical insights into malaria pathogenesis and aid disease interventions.

Var genes have a two-exon structure [4]. The first exon is large ( 3.5 to $9.0 \mathrm{~kb}$ ) and encodes multiple adhesion domains called the Duffy binding-like (DBL) and cysteine-rich interdomain region (CIDR). The second exon is smaller ( $\sim 1.0$ to $1.5 \mathrm{~kb}$ ) and codes for a more conserved cytoplasmic tail. Although PfEMP1 sequences are highly diverse, the adhesion domains can be grouped by sequence similarity [10] into seven types of DBL domains $(\alpha, \alpha, \beta, \gamma, \delta, \varepsilon$, and $\mathrm{x})$ and four types of CIDR domains $(\alpha$, $\alpha_{1}, \beta$, and $\gamma$ ) that have been used as criteria for dissecting PfEMP1 protein domain structures and binding functions.

The PfEMP1 proteins in the 3D7 genome have been arbitrarily classified into one of seventeen different protein architectural types based upon domain composition $[6,11]$ and divided into three major $(\mathrm{A}, \mathrm{B}$, and $\mathrm{C})$ and two intermediate $(\mathrm{B} / \mathrm{A}$ and $\mathrm{B} / \mathrm{C})$ groups on the basis of $5^{\prime}$ upstream (Ups) sequence and chromosomal location $[6,12,13]$. Var group A genes have UpsA flanking sequences and are located in sub-telomeric regions transcribed toward the telomere, while group B consists of telomeric var genes flanked by UpsB sequences that are transcribed toward the centromere, and group $\mathrm{C}$ are flanked by UpsC sequences and are located in central chromosomal regions. Group B/A genes are very similar in location and transcriptional orientation to group $B$ genes, but are located further from the telomere following other var genes or pseudogenes. In contrast, group $\mathrm{B} / \mathrm{C}$ genes have an UpsB-like 5' flanking sequence, but are located in central chromosomal regions. Thus, it has been postulated that groups $\mathrm{B} / \mathrm{A}$ and $\mathrm{B} / \mathrm{C}$ represent transitional groups between the major groupings [13].
Inter-isolate comparisons have also revealed the existence of three unusual genes: var1csa, var2csa, and Type 3 var genes, which appear in nearly all parasite isolates $[12,14$ 19]. These semi-conserved homologs may have important roles in the host-parasite interaction. The PfEMP1 encoded by var2csa binds the placental adhesion receptor, chondroitin sulfate A (CSA), and therefore has a critical role in the pathogenesis of pregnancy associated malaria $[20,21]$, while no function has yet been ascribed to the proteins encoded by var1csa and Type 3 var.

The genomic organization of var genes may have an important role in var gene evolution. Similar to other variant antigen families, gene recombination or gene conversion between var paralogs may contribute to the rapid evolution of the gene family [22-24]. It has been hypothesized that the frequency of recombination between var genes may depend upon chromosomal location, gene orientation, and homology in the gene flanking sequence. Sequence and binding analysis of 3D7 var genes indicate that groups $\mathrm{B}$ and $\mathrm{C}$ PfEMP1 proteins bind the primary microvasculature receptor (CD36) while group A PfEMP1 proteins do not $[12,13,25]$. Thus, var gene recombination hierarchies may promote the evolution of PfEMP1 adhesion groups with different patterns of sequestration and disease. A fundamental question is whether the gene organization observed in 3D7 occurs in other parasite isolates and contributes to a general recombination mechanism shaping the variant antigen repertoire.

To investigate evolutionary mechanisms of the var gene family and provide new tools to study the role of PfEMP1 proteins in mediating cytoadhesion, we have sequenced var genes from isolate IT4/25/4 (IT4), which has maintained the ability to cytoadhere after in vitro adaptation [26-29], and compared these genes to the var repertories of the 3D7 genome reference isolate and of the HB3 isolate, for which sequence contigs were recently made available (Plasmodium falciparum HB3 Sequencing Project, Broad Institute of Harvard and MIT [30]). Although there are currently relatively few reports, isolate HB3 also maintains cytoadherence in culture [31,32] and is therefore a useful addition to comparative var analyses.

All three parasites, IT4, HB3, and 3D7, have been cloned in vitro and represent single parasite genotypes. The IT4 parasite was originally isolated from Brazil [33], but is known to have undergone accidental cross-contamination at an early stage of its history after in vitro adaptation [34]. The HB3 clone was derived from the Honduras I/ CDC isolate [35] and the NF54 parent to the 3D7 clone was isolated from an individual who lived near an airport in Amsterdam and never left the Netherlands [36]. Based upon genotyping and parasite population studies, IT4 
groups with Asian isolates, 3D7 groups with African isolates, and HB3 represents Central America [37].

Despite progress in understanding the mechanisms of cytoadhesion and antigenic variation of PfEMP1, limited information about the factors regulating variant antigen diversification and the extent of repertoire overlap between parasite isolates exists. Most studies have relied on small var sequence "tags" amplified from the first DBL domain in PfEMP1 proteins [19,23,38-48]. The studies presented here represent the first comprehensive analyses of var genes across multiple parasite isolates. These comparisons reveal general principles of var gene organization that have become established across geographically diverse parasite isolates and provide powerful tools to study the cytoadherent and immunogenic properties of PfEMP1 proteins.

\section{Results}

The var gene repertoires from the IT4 and HB3 isolates

Conservation in the var gene $5^{\prime}$ and $3^{\prime}$ gene-flanking regions, the semi-conserved exon 2, and other domains allowed us to design a series of primers (Additional file 4: Table S1) and extend IT4 var tags that we had previously sequenced from the PfEMP1 DBL $\alpha, \beta, \gamma$, and $\delta$ domains [49]. We sequenced 28 full-length var genes and 10 partial genes from the IT4 isolate [GenBank:EF158071EF158105], in addition to the 10 full-length var genes that have been previously characterized (Figure 1). These genes represent all but 11 of the 59 IT4 sequence tags identified from other studies (Tables S2 and S3)[42,4951]. In order to estimate the proportion of IT4 var genes represented by these sequences, we searched the $1 \times$ coverage IT4 genome sequence at the Wellcome Trust Sanger Institute [52] for additional var sequences. Out of 949 reads with sequence similarity to the first exon of any known var genes, only $15 \%$ do not overlap with our data set. Assembly of these reads shows that most of the nonoverlapping reads represent small sequence fragments no larger than a single read and three partial gene fragments of 3-4 kb (data not shown). Thus, the var gene repertoire presented here includes partial or complete sequence for most IT4 var genes. Eight var genes were mapped to specific chromosomes using pulsed-field gel electrophoresis and Southern analysis (Figure 1, data not shown) and in some cases, intrachromosomal location (central versus sub-telomeric) was identified based on ApaI restriction fragment length [53]. The chromosomal locations of a further 13 var genes were based on previously published data [42].

Analysis of the HB3 sequence contigs obtained from the $10 \times$ coverage genome sequence at the Broad Institute[30] identified 52 var genes that contain a DBL $\alpha$ domain as well as two var2csa homologs; 39 of the 54 var genes are full-length, 9 are incomplete and six are pseudogenes containing stops or frame-shifts (Figure 2). Examination of $5^{\prime}$ and $3^{\prime}$ flanking sequences (see Materials and Methods) enabled us to predict the chromosomal location of most genes (Figure 2), although in a number of cases they could not be assigned to specific chromosome ends. These predictions assume that recombination has not changed the arrangement of chromosome ends in HB3 and 3D7.

Comparisons of the IT4 and HB3 PfEMP1 protein domain architecture revealed representatives of most classes previously described in 3D7 (Additional file 1) [6] plus fourteen new types (Figures 1 \&2). Of the 31 domain architectures, most types contain only a single representative per isolate and only seven $(1,5,7,8,11,13$, and 17) are found in all three isolates. While five $(2,6,9,14$, and $16)$ are found only in $3 D 7$, eight $(18-25)$ are unique to IT4, and six (26-31) to HB3 (Table 1). Moreover, the distribution of var genes among the shared domain architecture classes differs substantially between isolates. More than half $(40 / 62)$ of the 3D7 var genes have a Type 1 architecture, but this type of var gene is rarer in IT4 (12/ $48)$ and HB3 (20/54). Conversely, IT4 contains six Type 11 var genes compared to only one in 3D7 and HB3; while HB3 contains six representatives of Type 27, which is not present in either 3D7 or IT4. The differential abundance of individual PfEMP1 types between parasite isolates and the presence of new PfEMP1 types in isolates IT4 and HB3 indicate considerable inter-strain plasticity in the variant antigen repertoire. Despite these differences in gene repertoires, the previously described tandem domain associations (DBL $\alpha$-CIDR1, DBL $\beta-C 2$, and DBL $\delta$-CIDR non- $\alpha$ types) $[6,10]$ are consistently preserved, indicating the potential structural and functional significance of these domain relationships.

While seven protein architectural types are shared among the three isolates, most var genes have overall amino acid sequence identities of $<50 \%$ in individual domains (Additional file 2), even those within the same architectural type. However, three var genes (var1csa, var2csa, and Type 3 var) are highly conserved at the sequence level, with $>75 \%$ identity over multiple domains. Partial gene sequence tags for these three var genes have been amplified from many parasite isolates indicating their unusual conservation for the var gene family [19]. However, these isolate-transcendent members can have different copy numbers between parasite isolates. For instance, while 3D7 has three copies of the Type 3 var, we could amplify only one copy in IT4 and did not find any copies in the genomic sequence from HB3. In addition, HB3 contains two var2csa copies rather than one copy as in 3D7 and IT4. Although var1csa is present in all three parasites, it is a truncated pseudogene in 3D7 (first exon) and the second exon has a frameshift in the HB3 allele. Also, var1csa is 


\begin{tabular}{|c|c|c|c|c|c|c|c|c|c|c|c|c|c|c|c|c|}
\hline \multirow{2}{*}{$\begin{array}{c}\begin{array}{c}\text { IT4 gene } \\
\text { name }\end{array} \\
\text { IT4var3 }\end{array}$} & \multirow{2}{*}{$\begin{array}{c}\text { Alias } \\
\text { Type } 3 \text { var }\end{array}$} & \multirow{2}{*}{$\begin{array}{c}\text { Ups } \\
\mathrm{A} 1\end{array}$} & \multicolumn{9}{|c|}{ Extracellular Domain Structure (Predicted) } & \multirow{2}{*}{\begin{tabular}{|c|} 
ATS \\
exon2A1 \\
\end{tabular}} & \multirow{3}{*}{$\begin{array}{c}\text { Dom } \\
\text { Struc } \\
\text { Type } \\
3 \\
20\end{array}$} & \multirow{3}{*}{$\begin{array}{c}\text { Chr } \\
\text { Loc } \\
13 S T t\end{array}$} & \multirow{3}{*}{\begin{tabular}{|c|}
$\begin{array}{c}\text { Var } \\
\text { Group }\end{array}$ \\
A1 \\
A1ST
\end{tabular}} & \multirow[t]{2}{*}{ Function } \\
\hline & & & DBL1 $\alpha 1$ & $\mathrm{DBL} 2 \varepsilon$ & & & & & & & & & & & & \\
\hline IT4var9 & R29var & $\mathrm{A} 1$ & DBL1 $1 \alpha 1$ & CIDR1 $\gamma$ & $\mathrm{DBL} 2 \gamma$ & $\mathrm{DBL} 3 \varepsilon$ & DBL3 $\delta$ & CIDR2 $\beta$ & & & & & & & & Rosetting, CR1 \\
\hline IT4var60 & & $\mathrm{A} 1$ & DBL $1 \alpha 1$ & CIDR1 $1 \gamma$ & DBL2 $\gamma$ & DBL3 $\beta_{2}$ & $\mathrm{DB}\llcorner 4 \varepsilon$ & $\mathrm{DBL} 5 \varepsilon$ & & & & exon $2 \mathrm{~A} 1$ & 21 & 12 & $\mathrm{~A} 1$ & Rosetting \\
\hline IT4var64 & & $\mathrm{A} 1$ & DBL $1 \alpha 1$ & CIDR1 $\gamma$ & $\mathrm{DBL} 2 \beta$ & C2 & 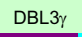 & $\mathrm{DBL} 4 \gamma$ & & & & exon $2 \mathrm{~A} 1$ & 19 & & $\mathrm{~A} 1$ & \\
\hline IT4var8 & & $\mathrm{A} 1$ & DBL $1 \alpha 1$ & CIDR1 $\gamma$ & $\mathrm{DBL} 2 \beta$ & $\mathrm{C} 2$ & DBL3 $\delta$ & CIDR2 $\gamma$ & $\mathrm{DBL} 4 \beta_{2}$ & $\mathrm{DBL} 5 \varepsilon$ & & exon $2 \mathrm{~A} 1$ & 23 & & $\mathrm{~A} 1$ & \\
\hline IT4var2 & & $\mathrm{A} 1$ & DBL $1 \alpha 1$ & CIDR1 $\gamma$ & DBL2 $\beta$ & $\mathrm{C} 2$ & 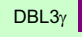 & DBL4 $\delta$ & CIDR2 $\beta$ & DBL5 $\beta$ & $\mathrm{C} 2$ & exon $2 \mathrm{~A} 1$ & 7 & & $\mathrm{~A} 1$ & \\
\hline IT4var22 & & $\mathrm{A} 1$ & DBL $1 \alpha 1$ & CIDR $1 \alpha 1$ & DBL2 $\beta$ & $\mathrm{C} 2$ & $\mathrm{DBL} 3 \gamma$ & $\mathrm{DBL} 4 \gamma$ & DBL5 & CIDR2 $\beta$ & & exon $2 \mathrm{~A} 1$ & 8 & & $\mathrm{~A} 1$ & \\
\hline IT4var7 & & $\mathrm{A} 1$ & DBL $1 \alpha 1$ & CIDR1 $\alpha 1$ & DBL2 $\beta$ & $\mathrm{C} 2$ & DBL3 $\beta$ & $\mathrm{C} 2$ & DBL $4 \gamma$ & DBL5 $\delta$ & CIDR2 $\beta$ & exon $2 \mathrm{~A} 1$ & 4 & & $\mathrm{~A} 1$ & \\
\hline IT4var18 & & $\mathrm{A} 1$ & DBL $1 \alpha 1$ & CIDR $1 \alpha 1$ & DBL2 $\beta$ & $\mathrm{C} 2$ & 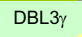 & $\mathrm{DBL} 4 \gamma$ & $\mathrm{C} 2$ & DBL5 $\delta$ & CIDR2 $\beta$ & exon $2 \mathrm{~A} 1$ & 22 & & $\mathrm{~A} 1$ & \\
\hline IT4var35 & var1csa & $\mathrm{A} 2$ & DBL $1 \alpha 1$ & CIDR $1 \alpha 1$ & DBL2 $\beta$ & $\mathrm{C} 2$ & $\mathrm{DBL} 3 \gamma$ & $\mathrm{DBL} 4 \varepsilon$ & DBL5 $\gamma$ & DBL6 $\beta$ & $\mathrm{DBL} 7 \varepsilon$ & & 17 & $10 \mathrm{~T}$ & $\mathrm{~A} 2$ & CSA, IgM \\
\hline IT4var54 & & B1 & DBL1 $\alpha$ & CIDR $1 \alpha$ & DBL2 $\delta$ & CIDR2 $\beta$ & & & & & & exon2B & 1 & & B1 & \\
\hline IT4var25 & & B1 & $\mathrm{DBL} 1 \alpha$ & CIDR $1 \alpha$ & DBL2 $\delta$ & CIDR2 $\beta$ & & & & & & exon2B & 1 & 8 or 9 & B1 & \\
\hline IT4var26 & & B1 & DBL1 $\alpha$ & CIDR $1 \alpha$ & DBL2 $\delta$ & CIDR2 $\beta$ & & & & & & exon2B & 1 & & B1 & \\
\hline IT4var33 & & B1 & $\mathrm{DBL} 1 \alpha$ & CIDR $1 \alpha$ & DBL2 $\delta$ & CIDR2 $\beta$ & & & & & & exon2B & 1 & 4 & B1 & \\
\hline IT4var29 & & B1 & DBL1 $\alpha$ & CIDR $1 \alpha$ & DBL28 & CIDR2 $\gamma$ & & & & & & exon2B & 1 & 1 & B1 & \\
\hline IT4var31 & A4tres & B1 & DBL1 $\alpha$ & CIDR $1 \alpha$ & DBL2 $\beta$ & $\mathrm{C} 2$ & 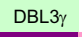 & & & & & & 15 & $1 \& 14 \mathrm{~T}$ & B1T & CD36, ICAM-1 \\
\hline IT4var12 & ItG var1 & B1 & DBL1 $\alpha$ & CIDR $1 \alpha$ & DBL2 $\beta$ & $\mathrm{C} 2$ & DBL3 $\delta$ & CIDR2 $\beta$ & & & & exon2B & 5 & $11 \mathrm{~T}$ & B1T & \\
\hline IT4var11 & & B1 & DBL1 $\alpha$ & CIDR $1 \alpha$ & DBL2 $\beta$ & C2 & DBL3 $\delta$ & CIDR2 $\beta$ & & & & exon2B & 5 & 7 & B1 & \\
\hline IT4var41 & & B1 & $\mathrm{DBL} 1 \alpha$ & CIDR $1 \alpha$ & $\mathrm{DBL} 2 \beta$ & $\mathrm{C} 2$ & DBL3y & DBL4 $\delta$ & CIDR2 $\beta$ & & & & $11 b$ & & B1 & \\
\hline IT4var17 & & B1 & DBL1 $\alpha$ & CIDR $1 \alpha$ & DBL2 $\beta$ & $\mathrm{C} 2$ & DBL3 $\delta$ & CIDR2 $\gamma$ & $\mathrm{DBL} 4 \gamma$ & & & exon2B & 25 & & B1 & \\
\hline IT4var14 & A4var & B1 & DBL1 $\alpha$ & CIDR $1 \alpha$ & DBL2 $\beta$ & $\mathrm{C} 2$ & DBL3 $\delta$ & CIDR2 $\gamma$ & $\mathrm{DBL} 4 \gamma$ & DBL5 $\beta$ & & & 18 & $13 \mathrm{Tc}$ & B1T & CD36, ICAM-1 \\
\hline IT4var13 & & B1 & DBL1 $\alpha$ & CIDR $1 \alpha$ & DBL2 $\beta$ & $\mathrm{C} 2$ & DBL2 $\delta *$ & CIDR2 $\gamma$ & $\mathrm{DBL} 4 \gamma$ & DBL5 $\beta 2$ & & exon2B & 18 & 4 & B1 & \\
\hline IT4var19 & & B1 & DBL1 $\alpha$ & CIDR1 $\alpha 1$ & DBL2 $\beta$ & $\mathrm{C} 2$ & 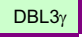 & DBL4 $\delta$ & CIDR2 $\beta$ & DBL5y & & exon2B & 24 & & B1 & \\
\hline IT4var44p & & B1 & DBL1 $\alpha$ & CIDR $1 \alpha$ & DBL2 $\beta$ & C2 & DBL38p & & & & & & u & & B1 & \\
\hline IT4var40p & & B1 & DBL1 $\alpha$ & CIDR $1 \alpha$ & DBL28p & & & & & & & & u & & B1 & \\
\hline IT4var45p & & B1 & DBL1 $\alpha$ & CIDR $1 \alpha$ & DBL28p & & & & & & & & u & & B1 & \\
\hline IT4var46p & & B1 & DBL1 $\alpha$ & CIDR $1 \alpha$ & DBL28p & & & & & & & & u & & B1 & \\
\hline IT4var16 & IT-ICAM1 & B2 & DBL1 $\alpha$ & CIDR $1 \alpha$ & DBL2 $\beta$ & $\mathrm{C} 2$ & $\mathrm{DBL} 3 \gamma$ & DBL4 & CIDR2 $\beta$ & & & & $11 b$ & 12 & B2 & CD36, ICAM-1 \\
\hline IT4var58p & & B3 & DBL1 $\alpha$ & CIDR1p & & & & & & & & & $\mathrm{u}$ & & B3 & \\
\hline IT4var15 & FCR3 var2 & B1 & DBL1 $\alpha$ & CIDR $1 \alpha$ & DBL2 $\beta$ & $\mathrm{C} 2$ & DBL3 $\delta$ & CIDR2 $\beta$ & & & & exon2B & 5 & $7 \mathrm{C}$ & $\mathrm{B} 1 \mathrm{C}$ & \\
\hline IT4var20 & FCR3 var3 & B2 & DBL1 $\alpha$ & CIDR1 $\alpha 1$ & DBL2 $\beta$ & $\mathrm{C} 2$ & $\mathrm{DBL} 3 \gamma$ & DBL3 $\delta$ & CIDR2 $\beta$ & & & exon2B & $11 a$ & $7 \mathrm{C}$ & $\mathrm{B} 2 \mathrm{C}$ & \\
\hline IT4var27 & & B3 & DBL1 $\alpha$ & CIDR $1 \alpha$ & DBL2 $\beta$ & $\mathrm{C} 2$ & $\mathrm{DBL} 3 \gamma$ & DBL4 $\delta$ & CIDR2 $\beta$ & & & exon2B & $11 \mathrm{~b}$ & $5-8 \mathrm{C}$ & B3C & Rosetting \\
\hline IT4var47 & & C1 & DBL1 $\alpha$ & CIDR $1 \alpha$ & DBL2 $\delta$ & CIDR2 $\beta$ & & & & & & exon2B & 1 & $4 \& 9$ & $\mathrm{C} 1$ & \\
\hline IT4var5 & & $\mathrm{C} 1$ & $\mathrm{DBL} 1 \alpha$ & CIDR $1 \alpha$ & DBL2 $\delta$ & CIDR2 $\beta$ & & & & & & exon2B & 1 & 13 & $\mathrm{C} 1$ & \\
\hline IT4var1 & & $\mathrm{C} 1$ & DBL1 $\alpha$ & CIDR $1 \alpha$ & DBL2 $\beta$ & $\mathrm{C} 2$ & $\mathrm{DBL} 3 \gamma$ & 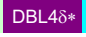 & CIDR2 $\gamma$ & & & & $11 \mathrm{c}$ & $5-8 \mathrm{C}$ & $\mathrm{C} 1 \mathrm{C}$ & Rosetting \\
\hline IT4var28 & & $\mathrm{C} 2$ & DBL1 $\alpha$ & CIDR $1 \alpha$ & DBL2 $\delta$ & CIDR2 $\beta$ & & & & & & exon2B & 1 & & $\mathrm{C} 2$ & \\
\hline IT4var23p & & $\mathrm{C} 1$ & DBL1 $1 \alpha$ & CIDR1p & & & & & & & & & $\mathrm{u}$ & & $\mathrm{C} 1$ & \\
\hline IT4var4 & var2csa & $\mathrm{E}$ & $\mathrm{DBL1}$ & DBL2 & DBL3 & $\mathrm{DBL} 4 \varepsilon$ & $\mathrm{DBL} 5 \varepsilon$ & DBL6 $\varepsilon$ & & & & exon2E & 13 & 12 & $\mathrm{E}$ & CSA, IgM \\
\hline IT4var39 & & & DBL1 $\alpha p$ & CIDR1 $\alpha$ & DBL2 $\delta$ & CIDR2 $\beta$ & & & & & & exon2B & 1 & & & \\
\hline IT4var36 & & & DBL1 $1 \alpha p$ & CIDR $1 \alpha$ & DBL2 $\delta$ & CIDR2 $\beta$ & & & & & & exon2B & 1 & & & \\
\hline IT4var21 & FCRS1.2 var1 & & DBL1 $\alpha$ & CIDR $1 \alpha$ & DBL2 $\delta$ & CIDR2 $\beta$ & & & & & & & 1 & 4 & & Rosetting, multi-adhesive \\
\hline IT4var24 & & & DBL1 $\alpha p$ & CIDR $1 \alpha$ & DBL2 $\delta$ & CIDR2 $\gamma$ & & & & & & exon2B & 1 & & & \\
\hline IT4var10 & varph17 & & DBL1 $\alpha$ & CIDR $1 \alpha$ & DBL2 $\beta$ & & & & & & & & u & & & \\
\hline IT4var32 & CS2 var & & $\mathrm{DBL} 1 \alpha$ & CIDR $1 \alpha$ & DBL2 $\gamma$ & DBL38 & CIDR2 $\beta$ & & & & & exon2B & 12 & 6 & & CSA (minor variant) \\
\hline IT4var6 & & & DBL1 $\alpha$ & CIDR1 $\alpha 1$ & DBL2 $\beta$ & $\mathrm{C} 2$ & 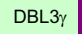 & DBL4 $4 \delta^{*}$ & CIDR2 $\beta$ & & & exon2B & $11 a$ & & & CSA (minor variant) \\
\hline IT4var30p & & & DBL1 $\alpha p$ & CIDR $1 \alpha$ & DBL28p & & & & & & & & u & & & \\
\hline IT4var51p & & & DBL1 $1 \alpha p$ & CIDR $1 \alpha$ & DBL28p & & & & & & & & u & & & \\
\hline IT4var34p & & & DBL1 $1 \alpha p$ & CIDR $1 \alpha$ & DBL2 $2 * \mathrm{p}$ & & & & & & & & $\mathrm{u}$ & & & \\
\hline
\end{tabular}

\section{Figure I}

Schematic representation of the IT4 var gene repertoire. Gene names, Ups sequence type, domain architecture, chromosomal location, transcription orientation, and binding functions are listed. IT4 var genes are primarily assigned to different groups on the basis of $5^{\prime}$ flanking sequence (Ups type) and chromosomal location when known. PfEMPI proteins are comprised of multiple domains termed $\mathrm{N}$-termimal segment (NTS), Duffy binding-like (DBL), cysteine-rich interdomain region (CIDR), $\mathrm{C} 2$, transmembrane (TM), and acidic terminal segment (ATS or exon2) which have been classified by sequence criteria into different types. The PfEMPI proteins in the 3D7 clone were arbitrarily classified into 17 different protein architectural types on the basis of domain composition [6]. Types 18-25 (bolded) are unique to IT4. Chromosome locations are indicated as T, ST, SST: first, second, and third var genes from the telomere respectively. C: internal var genes. t: transcribed towards telomere, c: transcribed towards centromere. The chromosomal location of var2csa was determined in [78]. Accession numbers for newly sequenced genes are EFI5807I-EFI58I05. 


\begin{tabular}{|c|c|c|c|c|c|c|c|c|c|c|c|c|c|c|c|c|c|}
\hline $\begin{array}{c}\text { HB3 gene } \\
\text { Name }\end{array}$ & $\begin{array}{c}\text { HB3 } \\
\text { Contig } \\
\text { Name }\end{array}$ & $\begin{array}{l}\text { Super } \\
\text { Contig }^{1}\end{array}$ & Alias & Ups & & Extrace & Ilular & Jomain & Structı & Ire (Pre & dicted) & & & ATS & $\begin{array}{l}\text { Dom } \\
\text { Struc } \\
\text { Type } \\
\end{array}$ & $\begin{array}{l}\text { Chr } \\
\text { Loc }\end{array}$ & $\begin{array}{c}\text { Var } \\
\text { Group }\end{array}$ \\
\hline HB3var6 & 699-3 & 1.47 & & $\mathrm{~A} 1$ & DBL1 $\alpha 1$ & CIDR1 $\gamma$ & DBL2 $\gamma$ & DBL3 $\delta$ & CIDR2 $\gamma$ & $\mathrm{DBL} 4 \varepsilon$ & DBL5 $\beta$ & $\mathrm{C} 2$ & $\mathrm{DBL} 6 \varepsilon$ & exon $2 \mathrm{~A} 1$ & 26 & $12-\mathrm{C} 2$ & $\mathrm{~A} 1 \mathrm{C}$ \\
\hline HB3var3 & 1737 & 1.265 & & $\mathrm{~A} 1$ & DBL1 $\alpha 1$ & CIDR1 $\alpha 1$ & DBL2 $\beta$ & $\mathrm{C} 2$ & DBL3 $\gamma$ & DBL4 $\delta$ & CIDR2 $\beta$ & DBL5 $\beta$ & $\mathrm{C} 2$ & & $7 \mathrm{~b}$ & $11 \mathrm{~L}-\mathrm{Tt}$ & $\mathrm{A} 1 \mathrm{~T}$ \\
\hline HB3var1 & $1000-2$ & 1.88 & & $\mathrm{~A} 1$ & DBL $1 \alpha 1$ & CIDR1 $1 \gamma 1$ & DBL2 $\beta$ & $\mathrm{C} 2$ & DBL3 $\gamma$ & $\mathrm{DBL} 4 \gamma$ & DBL5 $\delta$ & CIDR2 $\beta$ & & exon $2 A 1$ & $8 b$ & 9R-STt & A1ST \\
\hline HB3var46 $\Psi$ & 1278 & 1.138 & & $\mathrm{~A} 1$ & DBL $1 \alpha 1$ & CIDR1 $\gamma$ & $\mathrm{DBL} 2 \gamma$ & DBL3 $\delta$ & CIDR2 $\beta$ & DBL4 $\beta$ & $\mathrm{C} 2$ & & & & $10 a$ & 7L-STt & $\mathrm{A} 1 \mathrm{ST}$ \\
\hline HB3var2 & 1210 & 1.125 & & $\mathrm{~A} 1$ & DBL $1 \alpha 1$ & CIDR $1 \alpha 1$ & DBL2 $\gamma$ & DBL3 $\delta$ & CIDR2 $\beta$ & $\mathrm{DBL} 4 \beta$ & $\mathrm{C} 2$ & & & exon $2 \mathrm{~A} 1$ & $10 \mathrm{~b}$ & 7L-Tt & A1T \\
\hline HB3var4 & 1703 & 1.253 & & $\mathrm{~A} 1$ & DBL $1 \alpha 1$ & CIDR1 $\gamma$ & $\mathrm{DBL} 2 \gamma$ & DBL3 $\delta$ & CIDR2 $\beta$ & $\mathrm{DBL} 4 \gamma$ & DBL5 $\beta$ & $\mathrm{C} 2$ & DBL6 $\varepsilon$ & & 31 & STt & $\mathrm{A} 1 \mathrm{ST}$ \\
\hline HB3var5 & 1235 & 1.130 & & A2 & $\mathrm{DBL} 1 \alpha 1$ & CIDR1 $\gamma$ & $\mathrm{DBL} 2 \gamma$ & DBL38 & CIDR2 $\beta$ & DBL4 $\beta$ & $\mathrm{C} 2$ & & & & $10 a$ & $5 \mathrm{~L}-\mathrm{Tt}$ & $\mathrm{A} 2 \mathrm{~T}$ \\
\hline HB3var1csa & 1108 & 1.105 & var1csa & $\mathrm{A} 2$ & $\mathrm{DBL} 1 \alpha 1$ & CIDR1 $\alpha 1$ & DBL2 $\beta$ & $\mathrm{C} 2$ & DBL3 $\gamma$ & $\mathrm{DBL} 4 \varepsilon$ & DBL5 $\gamma$ & DBL6 $\beta$ & DBL7 $\varepsilon$ & & 17 & $\mathrm{STt}$ & A2ST \\
\hline HB3var7 & $1604-1$ & 1.226 & & B1 & DBL1 $\alpha$ & CIDR1 $\alpha$ & DBL2 $\delta$ & CIDR2 $\gamma$ & DBL3 $\gamma$ & DBL4 $\beta$ & $\mathrm{C} 2$ & DBL5 $\varepsilon$ & & & 30 & SSTC & B1SST \\
\hline HB3var14 & 1308 & 1.144 & & B1 & DBL1 $\alpha$ & CIDR1 $\alpha$ & DBL2 $\delta$ & CIDR2 $\beta$ & & & & & & exon2B & $1 \mathrm{a}$ & 10R-Tc & B1T \\
\hline HB3var16 & $1000-1$ & 1.188 & & B1 & DBL1 $\alpha$ & CIDR1 $\alpha$ & DBL2 $\delta$ & CIDR2 $\beta$ & & & & & & exon2B & $1 a$ & 9R-Tc & B1T \\
\hline HB3var18 & 1523 & 1.203 & & B1 & DBL1 $\alpha$ & CIDR1 $\alpha$ & DBL2 $\delta$ & CIDR2 $\beta$ & & & & & & exon2B & $1 \mathrm{a}$ & 11R-Tc & B1T \\
\hline HB3var12 & 1040 & 1.196 & & B1 & $\mathrm{DBL} 1 \alpha$ & CIDR $1 \alpha$ & DBL2 $\delta$ & CIDR2 $\beta$ & & & & & & exon2B & $1 a$ & 9L-Tc & B1T \\
\hline HB3var47\% & 1161 & 1.114 & & B1 & $\mathrm{DBL} 1 \alpha$ & CIDR $1 \alpha$ & DBL2 $\delta$ & CIDR2 $\beta p$ & & & & & & & $1 \mathrm{a}$ & $4 \mathrm{R}-\mathrm{Tc}$ & B1T \\
\hline HB3var13 & 1107 & 1.105 & & B1 & DBL1 $\alpha$ & CIDR $1 \alpha$ & DBL2 $\delta$ & CIDR2 $\beta$ & & & & & & & $1 a$ & Tc & B1T \\
\hline HB3var15 & 1426 & 1.171 & & B1 & $\mathrm{DBL} 1 \alpha$ & CIDR $1 \alpha$ & DBL2 $\delta$ & CIDR2 $\beta$ & & & & & & & $1 a$ & Tc & B1T \\
\hline HB3var20 & 1604-2 & 1.226 & & B1 & $\mathrm{DBL} 1 \alpha$ & CIDR $1 \alpha$ & DBL2 $\delta$ & CIDR2 $\beta$ & & & & & & exon2B & $1 \mathrm{a}$ & Tc & B1T \\
\hline HB3var11 & 1499 & 1.193 & & B1 & DBL1 $\alpha$ & CIDR1 $\alpha$ & DBL2 $\delta$ & CIDR2 $\beta$ & DBL3 $\varepsilon$ & & & & & exon2B & 14 & Tc & B1T \\
\hline HB3var10 & 1587 & 1.220 & & B1 & $\mathrm{DBL} 1 \alpha$ & CIDR1 $\alpha$ & DBL2 $\beta$ & $\mathrm{C} 2$ & DBL38 & CIDR2 $\beta$ & & & & & 5 & 13R-Tc & B1T \\
\hline HВ3var48\% & 1479 & 1.189 & & B1 & DBL1 $\alpha$ & CIDR1 $\alpha$ & DBL2 $\beta$ & $\mathrm{C} 2$ & DBL3 $\gamma$ & $\mathrm{DBL} 4 \beta$ & $\mathrm{C} 2$ & & & & u & Tc & B1T \\
\hline HB3var8 & 1334 & 1.153 & & B1 & $\mathrm{DBL} 1 \alpha$ & CIDR1 $\alpha$ & DBL2 $\delta$ & CIDR2 $\gamma$ & DBL3 $\gamma$ & DBL4 $\beta$ & C2 & DBL5 $\varepsilon$ & & exon2B & 30 & Tc & B1T \\
\hline HB3var9 & 1408 & 1.168 & & B1 & DBL1 $\alpha$ & CIDR $1 \alpha$ & DBL2 $\delta$ & CIDR2 $\gamma$ & DBL3 $\beta$ & C2 & DBL4 $4 \varepsilon$ & & & exon2B & $27 a$ & Tc & B1T \\
\hline HB3var494 & 1181 & 1.118 & & B1 & $\mathrm{DBL} 1 \alpha$ & CIDR1 $\alpha$ & DBL2 & CIDR2 $\gamma$ & DBL3 $\beta$ & C2 & $\mathrm{DBL} 4 \varepsilon$ & & & exon2B & $27 a$ & 12R-Tc & B1T \\
\hline HB3var38p & 1966 & 1.350 & & B1 & $\mathrm{DBL} 1 \alpha$ & CIDR $1 \alpha$ & & & & & & & & & $\mathrm{u}$ & Tc? & B1 \\
\hline HB3var39p & 2007 & 1.368 & & B1 & DBL1 $\alpha$ & CIDR $1 \alpha$ & & & & & & & & & $\mathrm{u}$ & 10L-Tc & B1T \\
\hline HB3 var37p & 2064 & 1.395 & & B1 & DBL1 $\alpha$ & CIDR1 $\alpha$ & DBL2 $\beta$ & $\mathrm{C} 2$ & DBL3 $\gamma$ & & & & & & $\mathrm{u}$ & & B1 \\
\hline HB3var19 & 1514 & 1.200 & & B1 & $\mathrm{DBL} 1 \alpha$ & CIDR1 $\alpha$ & DBL2 $\delta$ & CIDR2 $\gamma$ & & & & & & exon2B & $1 b$ & $12-\mathrm{C} 1$ & B1C \\
\hline HB3var17 & $1296-2$ & 1.142 & & B1 & $\mathrm{DBL} 1 \alpha$ & CIDR1 $\alpha$ & DBL2 $\beta$ & $\mathrm{C} 2$ & DBL3 $\gamma$ & DBL48 & CIDR2 $\beta$ & & & exon2B & $11 b$ & $7-C$ & B1C \\
\hline HB3var22 & $752-4$ & 1.54 & & B1 & DBL1 $\alpha$ & CIDR1 $\alpha$ & DBL2 $\delta$ & CIDR2 $\gamma$ & DBL3 $\varepsilon$ & $\mathrm{DBL} 4 \varepsilon$ & $\mathrm{DBL} 5 \varepsilon$ & & & exon2B & 28 & $8-C$ & B1C \\
\hline HB3var21 & 1671_1 & 1.243 & & B1 & $\mathrm{DBL} 1 \alpha$ & CIDR1 $\alpha$ & DBL2 $\beta$ & $\mathrm{C} 2$ & $\mathrm{DBL} 3 \gamma$ & DBL4 $\delta$ & CIDR2 $\gamma$ & DBL5 $\varepsilon$ & & exon2B & 29 & 7-C & B1C \\
\hline HB3var26 & 699-4 & 1.47 & & B3 & $\mathrm{DBL} 1 \alpha$ & CIDR $1 \alpha$ & DBL2 $\delta$ & CIDR2 $\beta$ & & & & & & exon2B & $1 a$ & $12-\mathrm{C} 2$ & B3C \\
\hline HB3var27 & $1074-1$ & 1.100 & & B3 & DBL1 $\alpha$ & CIDR $1 \alpha$ & DBL2 $\delta$ & CIDR2 $\beta$ & & & & & & exon2B & $1 \mathrm{a}$ & $4-\mathrm{C} 1$ & B3C \\
\hline HB3var24 & $752-2$ & 1.54 & & B3 & $\mathrm{DBL} 1 \alpha$ & CIDR $1 \alpha$ & DBL2 $\delta$ & CIDR2 $\gamma$ & DBL3 $\beta$ & $\mathrm{C} 2$ & $\mathrm{DBL} 4 \varepsilon$ & & & exon2B & $27 a$ & $8-C$ & B3C \\
\hline HB3var25 & $752-3$ & 1.54 & & B3 & $\mathrm{DBL} 1 \alpha$ & CIDR $1 \alpha$ & DBL2 $\delta$ & CIDR2 $\beta$ & DBL3 $\beta$ & $\mathrm{C} 2$ & $\mathrm{DBL} 4 \varepsilon$ & & & exon2B & $27 b$ & $8-C$ & B3C \\
\hline HB3var23 & 699-1 & 1.47 & & B4 & DBL1 $\alpha$ & CIDR1 $\alpha$ & DBL2 $\delta$ & CIDR2 $\gamma$ & DBL3 $\beta$ & $\mathrm{C} 2$ & DBL4 $\varepsilon$ & & & exon2B & $27 a$ & $12-\mathrm{C} 2$ & B4C \\
\hline HB3var28 & $1074-2$ & 1.100 & & C1 & $\mathrm{DBL} 1 \alpha$ & CIDR1 $\alpha$ & DBL2 $\delta$ & CIDR2 $\beta$ & & & & & & exon2B & $1 \mathrm{a}$ & $4-C 1$ & C1C \\
\hline HB3var29 & $1074-3$ & 1.100 & & C1 & $\mathrm{DBL} 1 \alpha$ & CIDR1 $\alpha$ & DBL2 $\delta$ & CIDR2 $\beta$ & & & & & & exon2B & $1 a$ & $4-C 1$ & C1C \\
\hline HB3var30 & 699-2 & 1.47 & & C1 & DBL1 $\alpha$ & CIDR1 $\alpha$ & DBL2 $\delta$ & CIDR2 $\beta$ & & & & & & exon2B & $1 a$ & $12-\mathrm{C} 2$ & C1C \\
\hline HB3var31 & 699-5 & 1.47 & & C1 & $\mathrm{DBL} 1 \alpha$ & CIDR1 $\alpha$ & DBL2 $\delta$ & CIDR2 $\beta$ & & & & & & exon2B & $1 a$ & $12-\mathrm{C} 2$ & C1C \\
\hline HB3var35 & $1296-3$ & 1.142 & & C1 & DBL1 $\alpha$ & CIDR $1 \alpha$ & DBL2 $\delta$ & CIDR2 $\beta$ & & & & & & exon2B & $1 a$ & 7-C & $\mathrm{C} 1 \mathrm{C}$ \\
\hline HB3var32 & 1459 & 1.183 & & C1 & $\mathrm{DBL} 1 \alpha$ & CIDR $1 \alpha$ & DBL2 $\delta$ & CIDR2 $\gamma$ & & & & & & exon $2 \mathrm{~A} 2$ & $1 b$ & $4-C 2$ & C1C \\
\hline HB3var33 & $752-5$ & 1.54 & & C1 & $\mathrm{DBL} 1 \alpha$ & CIDR $1 \alpha$ & DBL2 $\delta$ & CIDR2 $\gamma$ & & & & & & exon $2 \mathrm{~A} 2$ & $1 b$ & $8-C$ & C1C \\
\hline HB3var514 & 1671_2 & 1.243 & & & DBL1 $\alpha$ & CIDR $1 \alpha$ & DBL2 $\delta$ & CIDR2 $\gamma$ & & & & & & & $1 b$ & 7-C & C \\
\hline 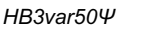 & $752 \_1$ & 1.54 & & C1 & $\mathrm{DBL} 1 \alpha$ & CIDR1 $\alpha$ & DBL2 $\delta$ & CIDR2 $\gamma$ & DBL3 $\beta$ & $\mathrm{C} 2$ & $\mathrm{DBL} 4 \varepsilon$ & & & & $27 a$ & $8-C$ & C1C \\
\hline HB3var34 & 209 & 1.8 & & C1 & $\mathrm{DBL} 1 \alpha$ & CIDR1 $\alpha$ & DBL2 $\beta$ & $\mathrm{C} 2$ & $\mathrm{DBL} 3 \gamma$ & DBL4 $\delta$ & CIDR2 $\beta$ & & & exon $2 \mathrm{~A} 2$ & $11 b$ & $6-C$ & $\mathrm{C} 1 \mathrm{C}$ \\
\hline HB3var36 & $1296-1$ & 1.142 & & $\mathrm{C} 2$ & DBL1 $\alpha$ & CIDR1 $\alpha$ & DBL2 $\delta$ & CIDR2 $\gamma$ & & & & & & & $1 \mathrm{~b}$ & $7-C$ & $\mathrm{C} 2 \mathrm{C}$ \\
\hline HB3var2csaA & 1727 & 1.262 & var2csaA & $E$ & DBL1 & DBL2 & DBL3 & $\mathrm{DBL} 4 \varepsilon$ & $\mathrm{DBL} 5 \varepsilon$ & DBL6 $\varepsilon$ & & & & exon2E & 13 & 12L-Tt & ET \\
\hline HB3var2csaB & 1817 & 1.295 & var2csaB & $\mathrm{E}$ & DBL1 & DBL2 & DBL3 & $\mathrm{DBL} 4 \varepsilon$ & $\mathrm{DBL} 5 \varepsilon$ & DBL6 $\varepsilon$ & & & & exon2E & 13 & $\mathrm{STt}$ & EST \\
\hline HB3var40p & 1678 & 1.245 & & & $\mathrm{DBL} 1 \alpha$ & CIDR1 $\alpha$ & DBL2 $\delta$ & CIDR2 $\gamma$ & DBL3 $\beta$ & $\mathrm{C} 2$ & DBL4 4 & & & exon2B & $27 a ?$ & 12R-STt? & \\
\hline HB3var41p & 2124 & 1.426 & & & $\mathrm{DBL} 1 \alpha$ & CIDR1 $\alpha$ & DBL2 $\delta$ & & & & & & & & $\mathrm{u}$ & & \\
\hline HB3var42p & 2463 & 1.684 & & & $\mathrm{DBL} 1 \alpha$ & CIDR $1 \alpha$ & & & & & & & & & $\mathrm{u}$ & & \\
\hline HB3var43p & 1941 & 1.337 & & & $\mathrm{DBL} 1 \alpha$ & & & & & & & & & & $\mathrm{u}$ & & \\
\hline HB3var44p & 2342 & 1.566 & & & $\mathrm{DBL} 1 \alpha$ & & & & & & & & & & $\mathrm{u}$ & & \\
\hline HB3var45p & 2622 & 1.841 & & & DBL1 $\alpha$ & & & & & & & & & & $\mathrm{u}$ & & \\
\hline
\end{tabular}

${ }^{1}$ supercontigs are given $\mathrm{NCBI}$ accession numbers that begin AANS01. For example supercontig 1.47 is AANS01000047 and supercontig 1.265 is AANS01000265.

\section{Figure 2}

Schematic representation of HB3 var genes. Genes are organized as in figure I and grouped according to 5 ' flanking sequence (Ups type) and chromosomal location. Partial $(p)$ and pseudogenes $(\Psi)$ are labeled. Bolded domain structure types are unique to the HB3 parasite line. Binding properties have not been mapped to HB3 PfEMPI proteins. 
Table I: Var gene chromosomal locations and domain architectures across isolates.

\begin{tabular}{|c|c|c|c|c|}
\hline \multirow[t]{2}{*}{ Ups group } & \multirow[t]{2}{*}{ Location } & \multicolumn{3}{|c|}{ Domain architecture } \\
\hline & & 3D7 & IT4 & HB3 \\
\hline \multirow[t]{2}{*}{ Al-2 } & $\mathrm{STt}$ & $2,3(3), 4,7,8^{a}, 9,10$ & $3,4,7,8,19,20,21,22,23$ & $\mathbf{7 , 8}, 10(1+\psi), 31$ \\
\hline & Cen & & & 26 \\
\hline \multirow[t]{2}{*}{$\mathrm{BI}$} & $\mathrm{Tc}, \mathrm{STc}$ & $\mathrm{I}(26), 2^{\mathrm{b}}, 6^{\mathrm{b}}, \mathrm{II}^{\mathrm{b}}, 12,14,16$ & $I(5), 5(3), I I, I 5, I 8(2), 24,25, P(4)$ & $\mathbf{I}(8+\psi), 5, \mathbf{I} \mathbf{I}, \mathrm{I} 4,27(\mathrm{I}+\psi), 30(2)^{\mathrm{b}}, \mathrm{P}(3+\psi)$ \\
\hline & Cen & 1 & & 28,29 \\
\hline \multirow[t]{2}{*}{ B2-4 } & Tc, STc & $\mathrm{I}, 2^{\mathrm{b}}$ & $\mathrm{II}(3), \mathrm{P}$ & \\
\hline & Cen & $I(2), 5, I 5$ & & $\mathrm{I}(2), 27(2+\psi)$ \\
\hline $\mathrm{Cl}$ & & I(II), 5 & $I(2), I I, P$ & I (7), II \\
\hline $\mathrm{C} 2$ & Cen & I & I & I \\
\hline $\mathrm{A} 2$ & $\mathrm{STt}$ & $\mathbf{1 7}(\psi)$ & 17 & 10,17 \\
\hline $\mathrm{E}$ & $\mathrm{STt}$ & 13 & 13 & $13(2)$ \\
\hline Unclassified & & & $I(4), I I, I 2, P(4)$ & $\mathrm{I}(\psi), 27, \mathrm{P}(6)$ \\
\hline Total & & $6 I+1 \psi$ & $48 c$ & $48+6 \psi^{c}$ \\
\hline
\end{tabular}

aBolded protein architectures were detected in all three isolates.

bThese genes were members of the B-ST var group.

cThe IT4 and HB3 are partial gene repertoire estimates.

believed to be truncated in many field isolates [18] and has a distinct gene transcription pattern from other var genes [54]. Therefore it may have a different biological role than other var genes.

Sequence comparison of $1.5-2.0 \mathrm{~kb}$ of $5^{\prime}$ flanking sequence from the 3D7 var genes has defined five upstream types; UpsA, B, C, D, and E types [6,11-13]. Phylogenetic analysis of $500 \mathrm{bp}$ of 5 ' flanking sequences from the IT4, HB3, and 3D7 var genes revealed that IT4 and HB3 have similar sequence groupings as 3D7 (Figure 3). While we found similar classes as in previous studies, we have sub-divided UpsB into four sub-groups (B1-B4) and UpsC into two sub-groups (C1 and C2). This study also revealed that UpsD is very similar to UpsA (Figure 3), and that these categories can be more accurately referred to as UpsA1 (formerly UpsA) and UpsA2 (formerly UpsD). Notably, the proportion of var genes in each Ups type is similar between isolates (Figures 1, 2, S1).

The 3D7 var gene repertoire has been previously categorized into three major $(\mathrm{A}, \mathrm{B}$, and $\mathrm{C})$ and two intermediate $(\mathrm{B} / \mathrm{A}$ and $\mathrm{B} / \mathrm{C})$ groups on the basis of Ups sequence and chromosomal location $[6,12,13]$. The HB3 and IT4 var genes can be similarly assigned to the three major groups on the basis of Ups sequence (Figure 3 ), but differences in chromosomal location between isolates argue for a modification of the sub-groupings. For example, the HB3 repertoire contains one UpsA1-associated var gene (HB3varG) that is in a central chromosomal cluster rather than the typical sub-telomeric location (Figure 4). Therefore, to allow for the future addition of 'atypical' genes, we have developed a naming system based upon var gene location and Ups sequence type. The Ups types (A1-2, B1-B4, C1-
2, and E) when known are listed first followed by a chromosome location reference. T, ST, and SST refer to the first, second, and third var genes from the telomere respectively and $\mathrm{C}$ refers to central var genes. For example, we have now separated var group A into sub-groups A1C, A1ST, and A1SST to represent central and sub-telomeric var genes, respectively. Similarly, group B is divided to represent var genes with corresponding central (B1C, B2C, etc.) or telomeric (B1T, B2T, etc.) locations. Members of the $\mathrm{B} /$ A group previously defined by Lavstsen et al. [13] are now classified as B1ST, B2ST, etc. denoting both the $5^{\prime}$ upstream type plus a distinct sub-telomeric chromosomal location which follows other var genes or pseudogenes [6].

As observed previously [55], the var gene chromosomal location was highly predictive of $5^{\prime}$ gene flanking sequence (Figure 4). For instance, nearly all centromeretranscribed var genes in the telomeric location were UpsB1 type (Figure 4). In contrast, members of the "transitional" B/C var group located in central chromosomal locations, associate with any of the 5 ' flanking sequences UpsB1-4. Interestingly, while HB3 contains a copy of the semi-conserved var1csa gene (domain architecture Type 17) with the expected UpsA2 sequence (formerly UpsD), the HB3 isolate is unique in having a second distinct PfEMP1 protein associated with the UpsA2 sequence (HB3var4, domain architecture Type 10). Thus, we have classified both within group A2ST. The highly conserved, and sequence divergent, var2csa remains in a separate Ups group (ET, EST, or ESST).

Although the general chromosomal distribution of var genes in sub-telomeric regions or central regions on chro- 


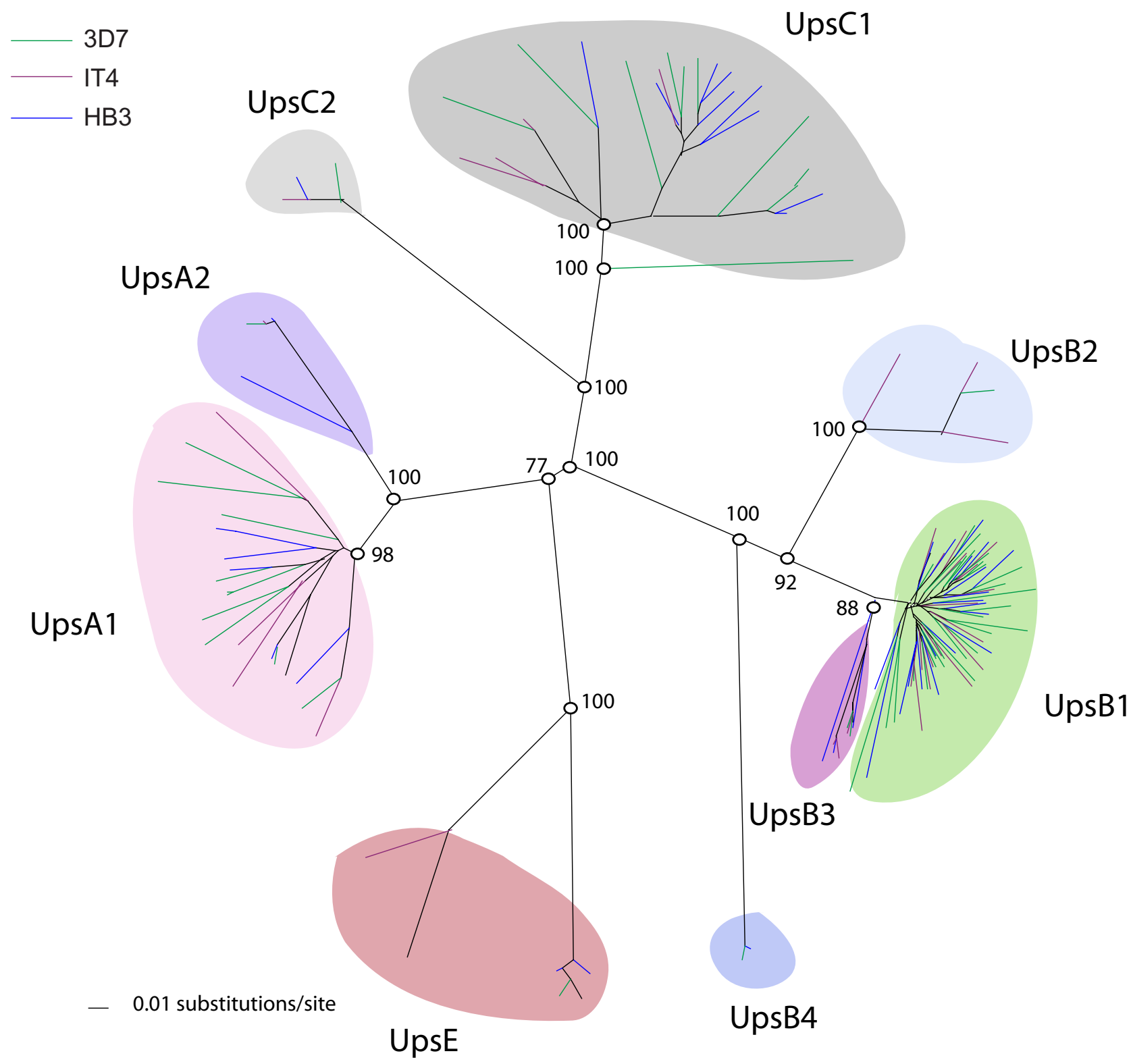

\section{Figure 3}

Phylogenetic comparison of var gene flanking regions from IT4, HB3, and 3D7 parasite isolates. A neighbor-joining tree was generated based upon 500 bp of 5 ' gene flanking sequence. Upstream groupings (Ups groups) with bootstrap support out of 1000 replicates are color shaded and labeled. Gene names have been removed from the figure for simplification. 
1
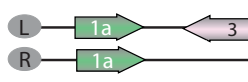

2

L -16

4

5

6

7

8

9

10

11

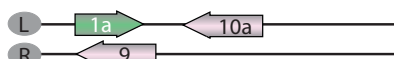

12

13

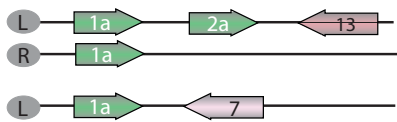

14
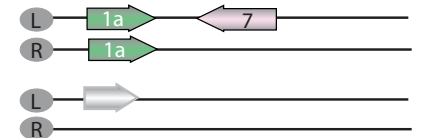

Central Vargenes:

4

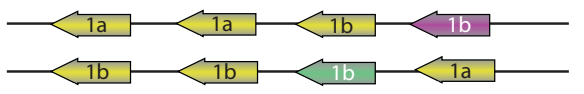

6

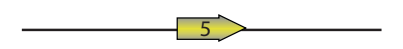

7

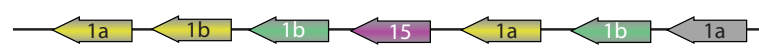

8

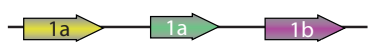

12

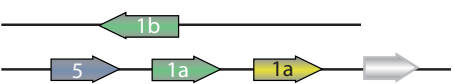

1

2

3

4

5

6

7

8

9

10

11

12

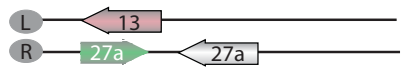

13

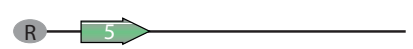

14

4

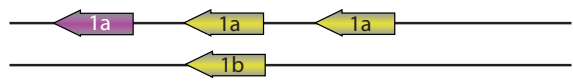

6

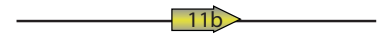

7

8

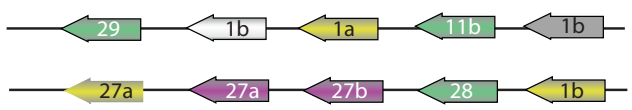

12

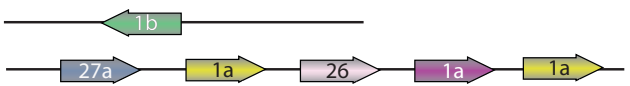

$\mathrm{C}_{1} \longrightarrow \mathrm{B}_{1} \longrightarrow \mathrm{B}_{3} \longrightarrow \mathrm{A}_{1} \longrightarrow \mathrm{E} \longrightarrow$

$\mathrm{C}_{2} \longrightarrow \mathrm{B} 2 \square \mathrm{B} 4 \square \mathrm{A} 2 \square \mathrm{U} \longrightarrow$

\section{Figure 4}

Chromosomal distribution of var genes in the 3D7 and HB3 parasite isolates. Var genes are color shaded according to 5 ' gene flanking Ups type ( $U$ indicates unknown) and labeled according to protein architecture. The chromosomal locations were predicted for 36 of the 54 HB3 PfEMPI proteins based upon gene flanking sequence and comparison to the 3D7 reference genome (see methods). Arrows without an outline indicate pseudogenes. 
mosomes $4,6,7,8$, and 12 are similar between the three isolates, the genes themselves are not conserved with the exception of var1csa, var2csa and Type 3 var. Significantly,var genes in the same chromosomal location from the three isolates differ in both sequence and protein architecture (Figure 4, Additional file 4: Table S4). Furthermore, the order of var Ups types in central var gene clusters differ between isolates (Figure 4). These differences between isolates are evidence of gene recombination that has occurred within the coding and geneflanking regions.

\section{Var gene recombination}

To study the genetic relationship of different var genes, we performed repertoire-wide nucleotide and amino acid sequence comparisons using a number of different approaches and visualization tools (see Materials and Methods). The Artemis Comparison Tool (ACT) [56] was used to visualize regions of similarity identified by reciprocal BLASTN searches of var exon 1 nucleotide sequences. Using criteria of a word size of 90nt, > 90\% identity we observed the gene duplication in 3D7 (PFD1235w and MAL8P1.207) and identified one gene duplication in HB3 (var $2 c s a A$ and var2csaB). These gene pairs are nearly identical over their entire lengths (Figure 5 \& Additional file 4: Table S5). These analyses also visualize the semi-conserved var genes (var1csa, var2csa and Type 3 var) identified above, which have multiple regions of high sequence similarity (> 90\%) between isolates (Additional file 4: Table S5).

ACT comparisons also identified several instances of partial sequence similarity (greater than $500 \mathrm{bp}$ ) between two var genes of the same isolate. Selected examples are shown (Table 2), illustrating the segmental nature of sequence similarity between genes, with only part of each sequence showing a high degree of sequence similarity to the other partner(s). The 3D7 repertoire contains four examples of such "chimeric" gene pairs, the current set of IT4 var genes has eight, and the HB3 repertoire has six (Table 2). In some cases (e.g. PFD0995c/PFD1000c/PFD1005c in 3D7), a var gene appears to be a "true" chimera of two different var genes (Figure 6), while in other cases the chimeras represent partial duplications between two var genes.

Despite geographic separation of 3D7, IT4, and 3D7, similar examples of segmental sequence similarity greater than 500 bp can be seen between var genes of the different isolates; with five examples between 3D7 and HB3, three between HB3 and IT4 and one between 3D7 and IT4 (Table 2). Remarkably, one of the inter-isolate gene pairs, HB3var23 and PFL1950w, are both the first var genes in a central cluster on chromosome 12 (Figure 4). These two genes have nearly identical and highly distinctive UpsB4 type 5 ' flanking sequences (Figure 3 ) and share approxi- mately 1000 bp of coding region identity (Table 2), but otherwise have diverged from one another. This region of similarity identifies a recombination event that likely predates the continental separation of $P$. falciparum isolates.

These analyses also demonstrate that most var genes share little sequence identity suggesting that the var genes have diverged extensively between parasite isolates and have undergone segmental recombination (Figure 5). However, the patterns of sequence identities are not random in that similarities preferentially occur between members of the same Ups group (Table 2). For instance, UpsA1 var genes are $7.3 \times$ more likely to share similarity with other UpsA1 genes than with different Ups groups and UpsB24 var genes are $8.6 \times$ more likely to share similarity within the UpsB2-4 group (Additional file 4: Table S6). The same trend holds for gene similarities involving smaller gene segments 90 nucleotides and up (Additional file 4: Table S6). An exception is central var genes, which contain "mixed" chimeras of UpsB and UpsC-associated var genes (Figure 6), suggesting both groups of central chromosome var genes are recombining.

To detect patterns of protein similarities, we conducted "repertoire-wide" dot-plot analyses using concatamers of var exon 1 sequences ordered by isolate and 5' flanking sequence type. These analyses are designed to detect small windows of sequence similarity ( $80 \%$ amino acid identity, 30 amino acid window length) between PfEMP1 amino acid sequences and clearly show that UpsA PfEMP1 proteins share less similarity with UpsB and UpsC proteins than with other UpsA proteins (Figure 7). Conversely, UpsB and UpsC proteins are indistinguishable in terms of their degree of sequence identity with each other. This analysis combined with the analyses of individual domains (Additional file 2) shows approximately as much overall repertoire similarity within as between these geographically diverse strains.

To identify the regions of similarity between PfEMP1 proteins, the dotplot matches were plotted along the length of individual proteins. Overall, the DBL1 domains in PfEMP1 proteins tend to have the most similarity between proteins, although there are regions of similarity in some CIDR domains (Additional file 3). Most of the similarity between PfEMP1 proteins, including between the B and C groups, is associated with semi-conserved homology blocks in DBL domains (Additional file 3). These homology blocks correspond to structural elements in solved structures $[57,58]$. These analyses also clearly illustrate that var2csa and Type 3 proteins share almost no identity with other PfEMP1 proteins. Curiously, the rosettingassociated IT4var60 protein is not related to other UpsA proteins over most of its length (data not shown). However, unlike the semi-conserved Type 3 var or var2csa, HB3 


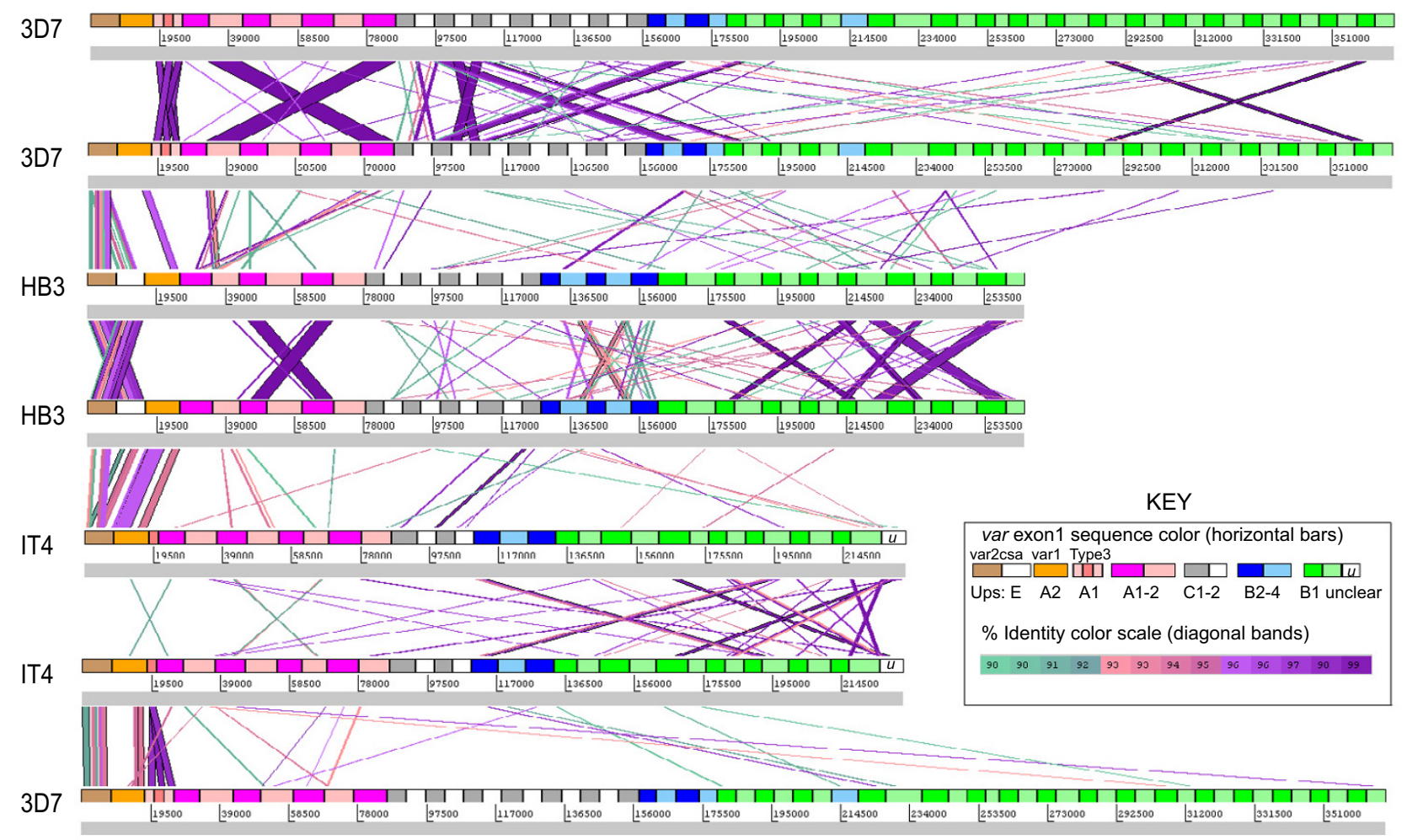

\section{Figure 5}

ACT nucleotide comparison of var gene repertoires. Concatamers of var gene exon I sequences were arranged sequentially by Ups type: UpsE, A, C, B2-4, and BI, (colored as indicated) with one genome per horizontal line. The isolate-transcendent var genes var2csa, varlcsa and Type 3 var are positioned at the left end of the concatemer. BLASTN was performed with word length set at 90 nucleotides (filter for low complexity removed). The comparisons were viewed in ACT with a window size of 120 nucleotides, at minimum $90 \%$ identity to show segments of similarity between var genes. Diagonal bands connecting individual var genes are colored according to percent identity, shown in the scale diagram (inset). Band width corresponds to region of sequence identity. Var names are listed, in order of appearance, in Additional file 4: Table S7.

and 3D7 do not have a var60 homolog. Although this result suggests that IT4var60 is not recombining with other var genes, more study is needed to determine its conservation in the parasite population. Taken together, these sequence comparisons support the hypothesis that var genes have differentiated into separately recombining groups that may be important to the evolution of the structure and function of PfEMP1 proteins.

\section{Discussion}

While the sequencing of the 3D7 genome has contributed greatly in determining PfEMP1 functions and genetic diversity [reviewed in [11]], the associations of var gene repertoires within and between parasite isolates and the factors regulating variant antigen diversification remain largely unknown. To gain understanding into the evolutionary mechanisms shaping the variant antigen repertoire we present here the nearly complete var repertoire of a cytoadhesive laboratory isolate, IT4/25/5, and compare it to the 3D7 genome reference isolate and the recently sequenced HB3 genome.

Despite the enormous diversity of these genes, several features of the var gene family are conserved across isolates including var groupings based upon central or telomeric chromosome location and 5 ' flanking sequence that may have an important role in the evolution and function of var genes. It has been hypothesized that an original ancestral var gene was duplicated and diverged into the three main var groups (A, B, and C) and subsequently into additional transitional groups [13]. This interpretation is supported by our analyses showing similar categories of var genes in all three parasite isolates.

Based upon sequence comparisons, B and C groups are more similar, even though these genes tend to occupy different chromosomal locations at sub-telomeric and central chromosomal regions, respectively. However, the 
Table 2: High scoring BLASTn matches within and between isolates.

\begin{tabular}{|c|c|c|c|c|c|c|}
\hline Comparison isolates & varl & var2 & Chromosome/group varl & Chromosome/group var2 & $\%$ ID & Match length (nt) \\
\hline \multicolumn{7}{|l|}{ between } \\
\hline$H B 3 \times 3 D 7$ & HB3var2 & MAL8PI.207a & 7/AIST & 8/AIST & 93.62 & $1 / 75$ \\
\hline$H B 3 \times 3 D 7$ & HB3var2 & $P F D / 235 w^{a}$ & 7/AIST & 4/AIST & 93.62 & $1 / 75$ \\
\hline HB3 $\times 3 D 7$ & HB3var4 & PFI3_0003 & AIST & I3/AIST & 91.19 & 636 \\
\hline HB3×3D7 & HB3var22 & PFII_052I & $8 / B I C$ & II/AIST & 92.62 & 664 \\
\hline HB3×3D7 & HB3var23 & PFL $1950 w$ & $12 / B 4 C^{b}$ & I2/B4C & 99.59 & 972 \\
\hline ITxHB3 & IT4var28 & HB3var35 & UpsC2 & $7 / \mathrm{CIC}$ & 99.34 & 1057 \\
\hline ITxHB3 & ITvar I6 & HB3var37(inc) & UpsB2 & UpsBI & 97 & 661 \\
\hline ITxHB3 & FCR3SI.2_I & HB3var27 & unknown & B3C & 99 & 1703 \\
\hline 3D7xIT & PFD0020c & IT4var6 & 4/AIST & unknown & 90 & 536 \\
\hline \multicolumn{7}{|l|}{ within } \\
\hline $3 D 7 \times 3 D 7 c$ & PFD0635w & PFD0630c & $4 / B 3 C$ & $4 / \mathrm{CIC}$ & 99 & 703 \\
\hline 3D7x3D7 & PFD0635w & PFD0630c & $4 / B 3 C$ & $4 / \mathrm{CIC}$ & 99.78 & 3645 \\
\hline 3D7x3D7 & PFD $1000 c$ & PFD0995c & $4 / \mathrm{CIC}$ & $4 / \mathrm{CIC}$ & 100 & 2831 \\
\hline 3D7×3D7 & PFD $1005 c$ & PFD I000c & $4 / B I C$ & $4 / \mathrm{CIC}$ & 99.92 & 2551 \\
\hline $3 \mathrm{D} 7 \times 3 \mathrm{D} 7$ & MALI $3 P I . I$ & PFE0005w & $|3 / \mathrm{B}| \mathrm{T}$ & $5 / \mathrm{BIT}$ & 100 & 2776 \\
\hline $\mathrm{HB} 3 \times \mathrm{HB} 3$ & HB3var3 & HB3var4 & II/AIST & AIST & 100 & 4948 \\
\hline HB3 $\times$ HB3 & HB3var23 & HB3var24 & I2/B3C & $8 / B 4 C$ & 94.52 & 1240 \\
\hline $\mathrm{HB} 3 \times \mathrm{HB} 3$ & HB3var23 & HB3var24 & I2/B3C & $8 / B 4 C$ & 93.12 & 567 \\
\hline $\mathrm{HB} 3 \times \mathrm{HB} 3$ & HB3var26 & HB3var23 & I2/B3C & I2/B3C & 96.56 & 552 \\
\hline $\mathrm{HB} 3 \times \mathrm{HB} 3$ & HB3var25 & HB3var24 & $8 / B 3 C$ & $8 / B 4 C$ & 92.86 & 518 \\
\hline $\mathrm{HB} 3 \times \mathrm{HB} 3$ & HB3var I5 (inc) & HB3var I 4 & $\mathrm{BIT}$ & IO/BIT & 100 & 788 \\
\hline $\mathrm{HB} 3 \times \mathrm{HB} 3$ & HB3var I5 (inc) & HB3var 14 & $\mathrm{BIT}$ & IO/BIT & 99.76 & 1657 \\
\hline $\mathrm{HB} 3 \times \mathrm{HB} 3$ & HB3var20 & HB3varl 4 & $\mathrm{BIT}$ & IO/BIT & 100 & 2577 \\
\hline $\mathrm{HB} 3 \times \mathrm{HB} 3$ & HB3var7 & HB3var8 & BIST & $\mathrm{BIT}$ & 99.86 & 5016 \\
\hline ITxIT & CS2 & IT4_var7 & 6/unknown & UpsAl & 99.67 & 905 \\
\hline ITxIT & IT4var4I & IT4_varl6 & UpsBI & 12/UpsB2 & 97.58 & 2359 \\
\hline ITxIT & CS2 & IT4_var19 & 6/unknown & UpsBI & 99.28 & 2221 \\
\hline ITxIT & CS2 & IT4_var29 & 6/unknown & I/UpsB| & 99.63 & 804 \\
\hline ITxIT & CS2 & IT4_var29 & 6/unknown & I/UpsBI & 93.69 & 697 \\
\hline ITxIT & CS2 & A4varICAM & 6/unknown & $|3 / \mathrm{B}| \mathrm{T}$ & 100 & 777 \\
\hline ITxIT & FCR3SI.2_I & IT4var58 & 4/unknown & unknown & 98 & 1046 \\
\hline ITxIT & IT4var23 & IT4var58 & unknown & unknown & 99 & 615 \\
\hline ITxIT & IT4var6 & IT4var 19 & unknown & UpsBI & 92 & 1348 \\
\hline
\end{tabular}

aMAL8PI.207 and PFD /235w are duplicated in 3D7, so the match with HB3var2 effectively occurs only once.

bThese two genes have a unique UpsB4 type 5' flanking sequence and are the first genes in a chromosome 12 central cluster in HB3 and 3D7, respectively.

cHighlighted rows indicate multiple hits of high sequence similarity, shared between the same gene pairs.

regions of similarity are predominantly associated with semiconserved homology blocks that are predicted to form the structural scaffolding for the DBL adhesion domains. Conversely, the group A genes differ greatly from the $\mathrm{B}$ and $\mathrm{C}$ groups while the coding region of the three isolate transcendent var genes, var1csa, var2csa, and Type 3 var genes have unique features and are different from all other var genes. However, these isolate transcendent var genes are more related to the UpsA group in that they are sub-telomeric, transcribed towards the telomere, and have 5 ' gene flanking regions that most resemble the UpsA type.

Repertoire-wide sequence comparisons show that most gene similarities occur between genes within the same var group, particularly for gene segments larger than $500 \mathrm{bp}$. An exception is central var clusters, which contain both UpsB and UpsC-associated var genes. While the functional significance of these different 5 ' promoter types is not completely understood [59], these two sets of central var genes appear to be recombining with each other. Taken together, these analyses suggest that var gene recombination preferentially occurs within var groups, with the exception of the semi-conserved var homologs that appear to recombine on their own. Further var gene comparisons of parasites undergoing more frequent recombination in nature or parasite crosses will be of interest to determine the relative frequency of intra- versus inter-group gene recombination. These findings provide insight into the mechanisms that generate antigenic diver- 


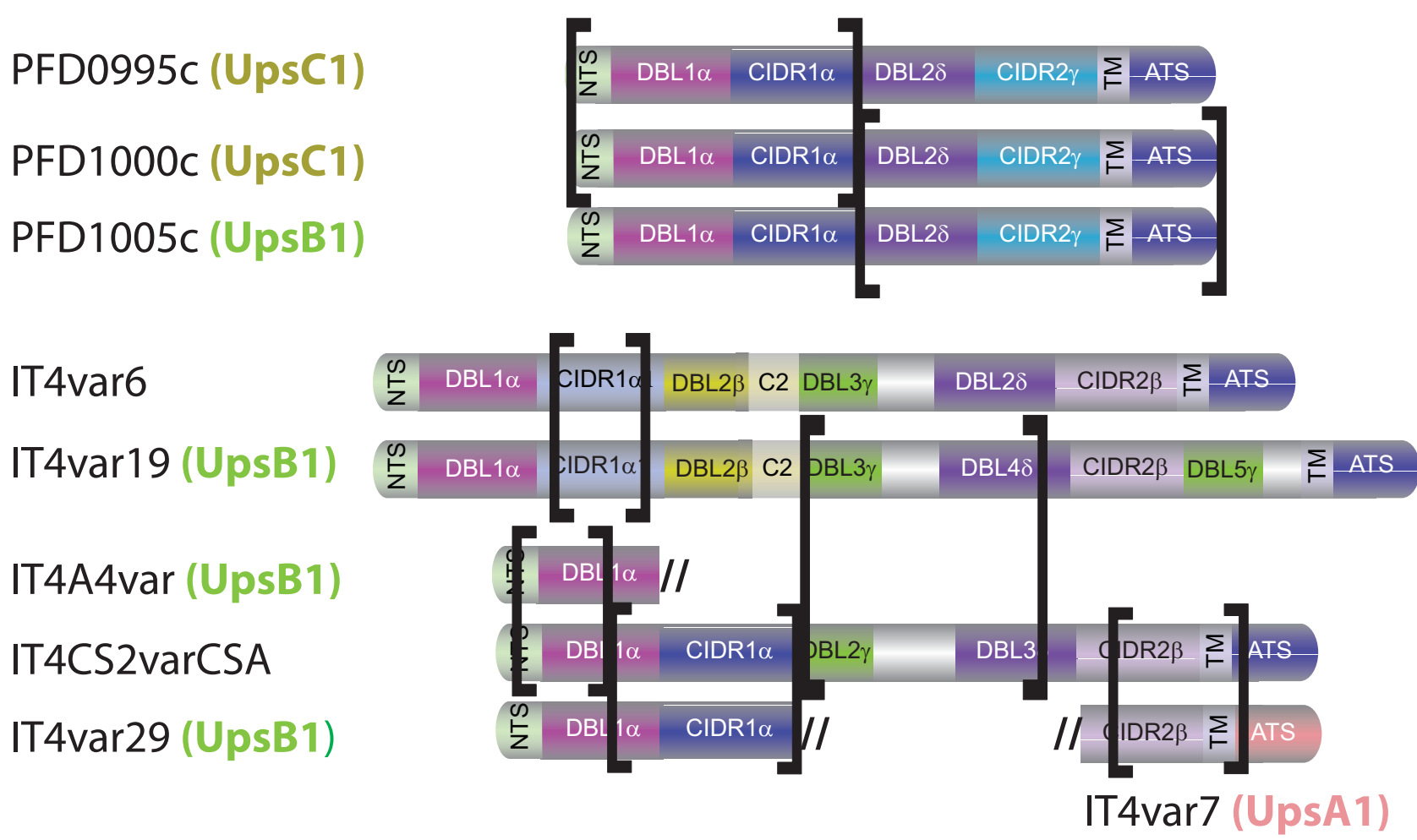

Figure 6

Examples of chimeric genes in the 3D7 and IT4 parasite isolates. Identical or nearly identical regions are indicated by brackets.

sity in $P$. falciparum through gene recombination hierarchies, and may have parallels in other variant antigen gene families in Plasmodium and other organisms.

The mechanisms of gene recombination/conversion are not well studied in $P$. falciparum. Sequence comparisons and restriction fragment length polymorphism analysis of parasite crosses and population studies suggest that both small ( 100-200 nt) and larger recombination events contribute to var gene evolution $[19,22,23]$. Here, we observe that chimeric junction sites are often not "clean" breakpoints and have smaller sections of 90-95\% identity 500 bp upstream and/or downstream of the central homologous region (data not shown). This feature may relate to a mechanism of recombination. Control of var gene expression has been connected to silence-inducing regulators of gene expression (e.g. Silent Information Regulator protein 2, SIR2) and chromatin packaging [60,61]. Recent studies have shown a possible link between factors involved in transcription regulation (including SIR2) and recombination (reviewed in $[62,63]$ ). It is interesting to speculate that in $P$. falciparum, factors that are silencing/ controlling var gene expression may also be involved in the recombination and gene conversion mechanisms.

From a study of 3D7 var genes expressed after antibody selection, it has been hypothesized that group A may contain common antigenic types that are responsible for severe disease [64]. Although the duplicated 3D7 UpsA var genes, PFD1235w and MAL8P1.207, have been proposed as a fourth isolate-transcendent variant, termed var4, our analyses do not support this conclusion since a var4 homolog was not found in IT4 or HB3, although the HB3 isolate contained a match over a portion of the gene. In addition, var4-like gene fragments were not common in a global survey of parasite isolates using gene-specific primers [19]. Instead, this observation may represent one of a number of between-genome var chimeras which are not present in all parasite genomes. More study is required to determine which segments of var4 are maintained in the parasite population and the extent to which the same segments are shared by different parasite isolates. 


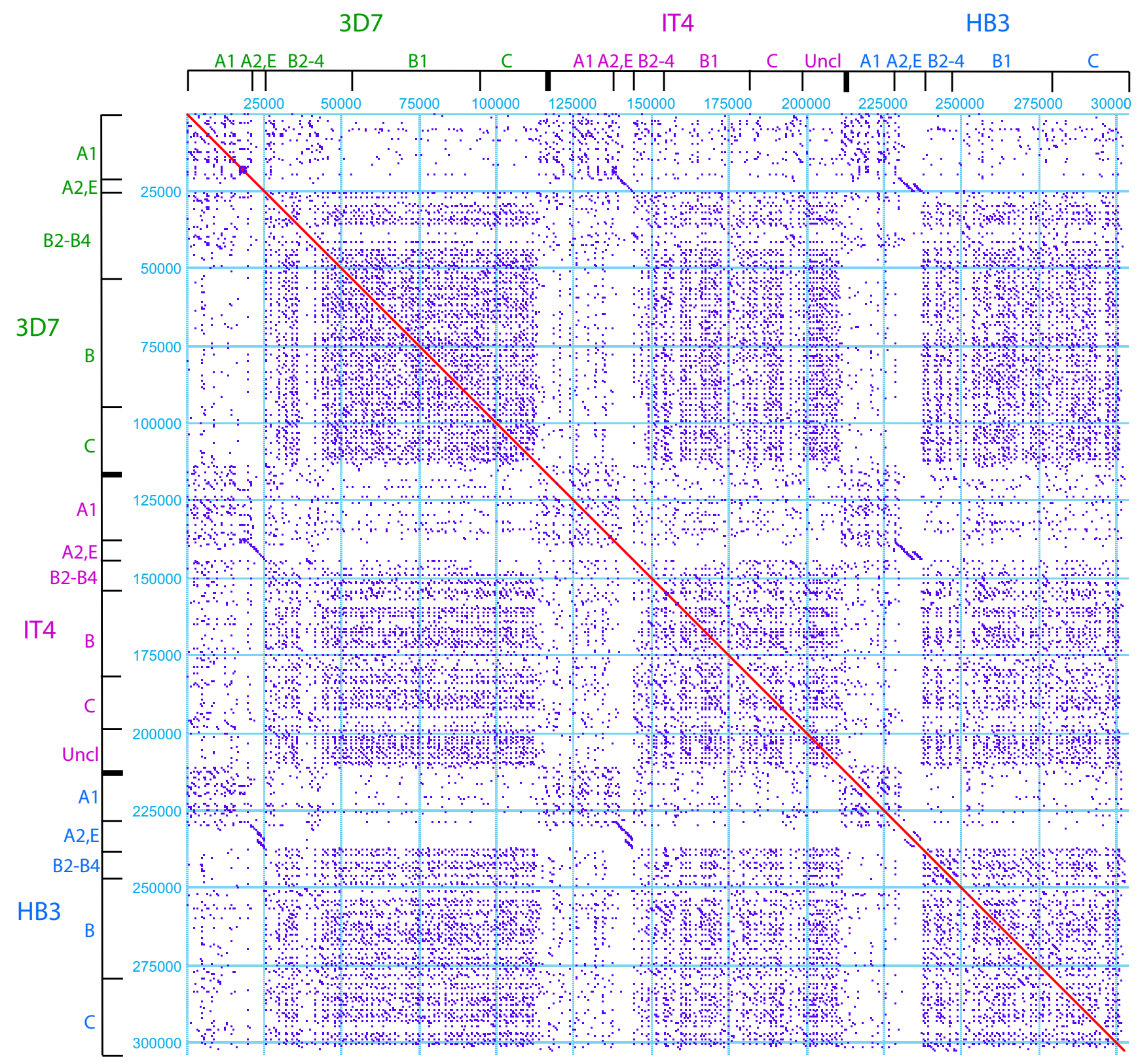

Figure 7

Dotplot comparisons of PfEMP I protein coding sequence. The extracellular binding region of PfEMPI proteins are organized by parasite isolate and 5' Ups sequence type. Uncl (unclassified) refers to sequences in which the Ups sequences have not been determined. Dot plot parameters include a window length of 30 amino acids and percent identity of $80 \%$ or greater. 
More generally, with the exception of the Type 3 var genes and var1csa, the UpsA-associated var genes are not highly conserved between the three isolates. This observation reinforces findings of high genetic diversity of UpsA-associated DBL $\alpha$ tags from a global collection of parasite isolates [19]. Various factors may influence the stability of large var gene segments across a parasite population, including malaria endemicity, the frequency of mixed infections, or functional selection on that gene segment for binding. The diversity of the UpsA var genes suggests that antibody cross-reactivity between different parasite isolates does not necessarily imply the presence of isolatetranscendent var genes, but may be due to cross-reacting antibody epitopes on different PfEMP1 sequences. Although the possibility that a subset of var genes may be associated with severe malaria remains, these genes may not be as conserved across parasite isolates as the pregnancy malaria vaccine candidate var2csa.

The concept of a recombination hierarchy has implications for the evolution of parasite virulence and disease investigation. The conservation of var groupings across isolates raises the possibility that var groups may be diverging and/or evolving in characteristic patterns. For instance, group A var genes, with the exception of the Type 3 var genes, encode larger proteins with more complex domain compositions and have different protein head structures (DBL1-CIDR1 domains) from other var groups (Figures 1 \&2). In contrast, the relatively small Type 1 proteins, which consist of four adhesion domains, are not associated with group A in any of the isolates. It has been suggested that immune selection can cause polymorphic antigens to self-organize into sets of non-overlapping variants within a population [65]. Increased frequency of inter-locus recombination or gene conversion may also act as a homogenizing force leading to the functional and structural specialization of different gene groups [25]. Interestingly, the proportion of small to larger PfEMP1 proteins and the distribution of PfEMP1 architectural types differed between isolates. Given the different selective pressures for binding and immune evasion, it may be to the parasite's advantage to have different sets of recombining genes $[66,67]$. These sets might include genes optimized to promote rapid parasite growth and transmission in the non-immune host, diversified genes that promote parasite transmission and persistence of infection in the face of higher levels of host immunity or organ-specific variants that expand parasite tropism to new host tissues, such as the placenta.

Unlike many isolates that have been adapted to in vitro cultivation, the IT4 genotype stably maintains the cytoadherent phenotype and therefore has become the primary model for this virulence determinant. CD36 binding, intercellular adhesion molecule 1 (ICAM-1) binding, and infected erythrocyte rosetting, or the binding of infected erythrocytes to uninfected erythrocytes, have been shown to reside in multiple different IT4 PfEMP1 proteins [for review, see [68]]. Although proteins that bind the same host receptor frequently use the same type of binding domain [11], the overall protein architectures are highly distinct. For instance, three ICAM-1 binding PfEMP1 proteins (A4tres, A4var, and IT-ICAM-1) all use DBL $\beta$ c2 domains but have different domain structure types and two rosetting PfEMP1 proteins, R29var [51] and FCRS1.2var1 [51,69] also have different domain structure types and bind different receptors on the erythrocyte surface. Our study completes the sequences for three additional IT4 var genes upregulated in rosetting parasite clones, which were previously identified by only their DBL $\alpha$ tag sequences (IT4var1, IT4var27, and IT4var60) [70]. Overall, the rosetting var protein structures are not highly related (Figure 1 ), although the DBL $1 \alpha_{1}$ domain is similar between the UpsA-linked R29var and IT4var60 predicted proteins, which may be significant because DBL1 $\alpha_{1}$ is an erythrocyte binding region in R29var [51]. In contrast, the other three rosetting-linked var genes (IT4var27, IT4var1, and FCRS1.2var1) have DBL $\alpha$ domains instead of $\mathrm{DBL} \alpha_{1}$ domains and associate with Ups B, C, or unknown Ups sequence. While these comparisons suggest that rosetting PfEMP1 proteins are not restricted to particular var groups, further study is needed to determine whether rosetting proteins in the same var group use a similar constellation of erythrocyte receptors.

\section{Conclusion}

A detailed understanding of the molecular mechanisms responsible for malaria pathogenesis is lacking, partly because of the complexity of the var gene family and the inability to model cytoadhesion with most cultureadapted laboratory isolates. In this study, we determined the var gene repertoires from the IT4 and HB3 isolates and provide evidence for a recombination hierarchy that shapes the evolution of the PfEMP1 virulence determinant. Furthermore, determination of the nearly complete var gene repertoire from the cytoadhesive IT4 parasite genotype, which has been adapted to both grow in the laboratory and infect new world monkeys, provides a unique capability to model cytoadhesion and immune acquisition in vitro and in vivo. Future binding and expression studies with cytoadhesive laboratory isolates, such as IT4 and HB3, in conjunction with analyses of the fully sequenced genomes will allow us to classify PfEMP1 proteins into biologically meaningful subsets and greatly accelerate understanding into malaria pathogenesis and immune evasion. 


\section{Methods \\ Parasites}

Var genes were cloned from genomic DNA of the A4 clonal line. The A4 clone was originally derived by micromanipulation from $P$. falciparum isolate IT4/25/5 [29]. The IT4/25/5 isolate is one of several isolates including FVO, FCR3, and Palo Alto that appear to have a common genetic origin due to a laboratory cross-contamination event [34].

\section{Long PCR amplification of var gene sequences}

Larger var gene PCR products were amplified from genomic DNA using previously described techniques [49]. PCR primers (Additional file 4: Table S1) were designed to the different types of var gene flanking sequence or the relatively conserved var exon 2. These primers were paired with gene-specific primers from small sequence tags that had been amplified from internal domains in IT4 PfEMP1 proteins [49]. PCR reactions were done using TaKaRa LA Taq ${ }^{\mathrm{TM}}$ polymerase (Fisher) following the manufacturer's recommendations and supplied buffer. $50 \mathrm{ul}$ reactions containing $50 \mathrm{ng}$ template, $1 \times$ buffer, $0.4 \mathrm{mM}$ dNTPs (each), $2.5 \mathrm{mM} \mathrm{MgCl}_{2}, 0.5 \mu \mathrm{M}$ primers, and $2.5 \mathrm{U}$ enzyme were run in a DNA Engine Dyad $^{\mathrm{TM}}$ Peltier Thermal Cycler from MJ Research. PCR conditions were 1 cycle of $94^{\circ} \mathrm{C}$ for $1 \mathrm{~min}$ followed by 35 cycles of $98^{\circ} \mathrm{C}$ for $1 \mathrm{~min}$, primer annealing temperature for $1 \mathrm{~min}$, and an extension temperature of $62-68^{\circ} \mathrm{C}$ for 8-18 min. Primer annealing temperatures were 0-5 degrees lower the TMs listed in Additional file 4: Table S1. Sequencing was performed on PCR products that were directly hydosheared and cloned into a sequencing vector or were first cloned into the $\mathrm{pCR}^{\circledR} 4$-TOPO vector from Invitrogen before hydoshearing and subcloning into the sequencing vector. Sequences were assembled using the PHRED/PHRAP/CONSED software suite [71]. To confirm that recombination had not occurred during the PCR reaction or bacterial cloning, specific oligos were designed along the length of var genes and independent PCR reactions were performed on genomic DNA.

\section{Var gene chromosome assignation by pulsed field gel}

IT4/FCR3 parasites were suspended in agarose blocks, then the intact chromosomes were size-fractionated on pulsed-field gels as described [72]. Gels were depurinated for $10 \mathrm{~min}$ in $0.25 \mathrm{M} \mathrm{HCl}$, rinsed, then capillary transferred to Hybond $\mathrm{N}+$ (GE/Amersham) in $0.4 \mathrm{M} \mathrm{NaOH}$. Blots were hybridized at $50^{\circ} \mathrm{C}$ with DBL $\alpha$ tag probes corresponding to var genes IT4var1,IT4var5, IT4var25, IT4var27,IT4var 29,IT4var33, IT4var60 and A4Tres, as detailed previously [73]. Chromosome-central location of IT4var1 and IT4var27 was confirmed by ApaI digestion and size separation on pulsed-field gels, with hybridization to these tags at $60^{\circ} \mathrm{C}$. Subtelomeric var genes lie on relatively short ApaI fragments [53], and these two genes are on large ( $>400 \mathrm{kbp}$ ) ApaI fragments.

\section{Extraction of var gene sequences from public genome project information}

Var genes were identified in HB3 contigs downloaded from the Plasmodium falciparum HB3 sequencing project, Broad Institute of Harvard and MIT [30]. Contig assembly 1 was used, which contains approximately $10 \times$ genomic coverage. To identify var genes, sequences were searched for a common DBL $\alpha$ motif, DIGDI, using Artemis genome viewer (Rutherford et al 2000). Var genes retrieved in this manner were confirmed by comparison to results from BLASTN with the full DBL1 $\alpha$ sequence of PFA0005w, at the Broad Institute malaria website. HB3 homologs for var2csa were identified by BLASTN search at the same website, using the 3D7 allele. HB3 pseudovar genes were not confirmed by reamplification, but had approximately the same level of sequence coverage as other var genes (8$10 \times)$. Where possible, the var gene chromosomal context was also noted, using \%GC content graphs to locate both telomeres and GC-rich DNA elements, which are short sequences associated only with central var genes [74]. The predicted HB3 chromosomal assignments were based upon comparison to the 3D7 isolate using the NUCmer program in MUMmer to identify sequence identities in var gene flanking regions [75]. Sequence data for $P$. falciparum 3D7 var genes and unassembled IT4 var reads were obtained from The Sanger Institute website [52]. Sequencing of $P$. falciparum IT4 is a component of the BioMalPar Consortium. For the ACT comparisons, two new 3D7 var genes are included, MAL7P1.212 and MAL8P1.220, which appear in the latest annotation [52]. These are both the most common type, Type 1, with UpsB type promoters, bringing the total number of this type in 3D7 up to 40 (out of 61 var genes).

\section{Sequence analysis}

PfEMP1 domain classification was performed according to previous criteria [10]. Neighbor-joining trees for all of the domains and flanking regions were generated using ClustalX for multiple alignments and PAUP*4.0b10 (* Phylogenetic Analysis Using Parsimony and other methods) [76]. Bootstrap analysis was performed with 1000 replicates. Gap opening and gap extension penalties of 5.0 and 0.05 or 10 and 0.1 were used for amino acid and DNA alignments, respectively. Percentage sequence identities of DBL, CIDR, and C2 domains were calculated using the algorithm in DNAStar MEGALIGN, version 5.0 based upon a ClustalW alignment. Means and standard deviations of these percentages were calculated and plotted in Excel.

Dotplot analysis was performed on concatemers of the variable extracellular region (exon1) sequences ordered 
by isolate and Ups type using the programs Megalign and DSGene. A percent identity matrix was used for all parameters tested (window length and percent identity threshold). To visualize the alignment results at the level of individual proteins, the output alignments from the dotplot were collected, and for each alignment, the aa positions of the alignment and Ups category of the pair of proteins were determined. For each aa position, the number of "alignment hits" from each Ups category was counted and plotted along the length of individual proteins. Microsoft Excel was used to generate plots of the number of hits from 3D7 PfEMP1 proteins of the different Ups types along the length of individual proteins for both IT4 and 3D7 PfEMP1 proteins. Based upon the distribution of var genes in the 3D7 isolate, the maximum number of hits at individual amino acid positions for genes of each promoter type is UpsA (9), UpsB (22), UpsC (13), UpsA2 (formerly UpsD; 1), UpsE (1), and UpsB2-4 (13, based upon previous published 2000 bp tree) $[6,12]$.

The Artemis Comparison Tool (ACT) was used to view exon1 for IT4, 3D7 and HB3 var genes. For each genome, a concatemer of var exon1 nucleotide sequences (from the start ATG to the splice donor site) was created. Sequences were organized by Ups group, and a string of $30 \mathrm{~N}$ s placed between each exon 1 pair to clarify gene borders. Local BLASTN, (word length 90 nucleotides, filter for low complexity removed) was performed in all possible combinations between IT, 3D7 and HB3 var exon 1 concatemers. These comparisons were then viewed with ACT $[56,77]$, with different window sizes (90 to 510 nucleotides), $90 \%$ minimum identity, and self-matches removed.

\section{Authors' contributions}

SMK carried out IT4 var gene sequencing, performed dotplot, phylogenetic, and other sequence analyses, and contributed to project design and coordination. SAK extracted var gene sequences from the HB3 public database and performed pulse-field gel analysis of IT4 var genes, ACT and sequence analysis of var genes. GA developed software to display dotplot information of individual proteins. ALS, SON, LMS, and WW amplified and sequenced IT4 var genes and performed PCR confirmations on genomic DNA. EL contributed to sequence analyses and ZC performed pulse-field gel analysis. CIN helped coordinate sequence analysis of var genes. PJM helped coordinate sequence analysis of var genes and performed the MUMmer analysis to assign HB3 var genes to chromosomes. JDS conceived the study, performed sequence analysis of var genes, and contributed to the projects design and coordination. SMK, SAK, CIN, PJM, and JDS wrote the manuscript. All authors approved the final manuscript.

\section{Additional material}

\section{Additional file 4 \\ Tables S1-S7 \\ Click here for file \\ [http://www.biomedcentral.com/content/supplementary/1471- \\ 2164-8-45-S4.pdf]}

\section{Additional file 1}

Schematic representation of $3 D 7$ var genes. Genes are organized as in Figures 1 and 2.

Click here for file

[http://www.biomedcentral.com/content/supplementary/1471-

2164-8-45-S1.pdf]

\section{Additional file 2}

Amino acid identity of PfEMP1 adhesion domains from 3D7 and IT4 parasite isolates. Pair-wise comparisons were performed for all of the adhesion domains in 3D7 and IT4 PfEMP1 proteins. For each individual domain, the average identity to all other IT4 or 3D7 domains of that type was determined. These means were then averaged for 3D7 and IT4. Error bars represent one standard deviation of the mean of means.

Click here for file

[http://www.biomedcentral.com/content/supplementary/14712164-8-45-S2.pdf]

\section{Additional file 3}

Amino acid identity between PfEMP1 proteins of different Ups types. Alignment "hits" collected from the dot plot shown in Figure 7 were plotted per amino acid position of individual proteins. Graphs represent 3D7 PfEMP1 protein alignment "hits" (including the var1csa pseudo-gene) plotted against individual IT4 or 3D7 PfEMP1 proteins. Based upon the distribution of var genes in the $3 D 7$ isolate, the maximum number of hits at individual amino acid positions for genes of each promoter type is UpsA (9), UpsB (22), UpsC (13), UpsA2 (formerly UpsD; 1), UpsE (1), and Ups B2-4 (13) $[6,11]$. Previously defined DBL homology blocks B, D and $H$ are labeled [10].

Click here for file

[http://www.biomedcentral.com/content/supplementary/14712164-8-45-S3.pdf]

\section{Acknowledgements}

We wish to thank Atashi Anupama, Dhileep Savam, Andy Leonard and Eric Flamoe for aid in script writing and Ellen Sisk, Trevor Hopkins, and Chad Austin for technical assistance. This work was supported by Bill \& Melinda Gates Foundation (JDS), National Institute of Health (grant ROI Al47953, JDS), and the MJ Murdock Charitable Trust (Seattle Biomedical Research Institute). We wish to acknowledge the Computational Biology Research Group, Medical Sciences Division, Oxford and the Bioinformatics core at SBRI for use of their services in this project. Sequence data were obtained from the Plasmodium falciparum HB3 Sequencing Project, funded by the NIAID, which was performed at Broad Institute of Harvard and MIT [30]. Sequence data for $P$. falciparum 3D7 and IT4 var genes was obtained from The Sanger Institute website [52]. Sequencing of $P$. falciparum 3D7 was accomplished as part of the Malaria Genome Project. Sequencing of $P$. falciparum IT4 is a component of the BioMalPar Consortium (European Union 6th Framework Program, Contract number LSHP-LT- 2004-503578). 


\section{References}

I. Snow RW, Guerra CA, Noor AM, Myint HY, Hay SI: The global distribution of clinical episodes of Plasmodium falciparum malaria. Nature 2005, 434:214-2I7.

2. Miller LH, Baruch DI, Marsh K, Doumbo OK: The pathogenic basis of malaria. Nature 2002, 4I5:673-9.

3. Baruch DI, Pasloske BL, Singh HB, Bi X, Ma XC, Feldman M, Tarasch TF, Howard RJ: Cloning the $P$. falciparum gene encoding PfEMPI, a malarial variant antigen and adherence receptor on the surface of parasitized human erythrocytes. Cell 1995 , 82:77-87.

4. Su XZ, Heatwole VM, Wertheimer SP, Guinet F, Herrfeldt JA, Peterson DS, Ravetch JA, Wellems TE: The large diverse gene family var encodes proteins involved in cytoadherence and antigenic variation of Plasmodium falciparum-infected erythrocytes. Cell 1995, 82:89-100.

5. Smith JD, Chitnis CE, Craig AG, Roberts DJ, Hudson-Taylor DE Peterson DS, Pinches R, Newbold $\mathrm{Cl}$, Miller LH: Switches in expression of Plasmodium falciparum var genes correlate with changes in antigenic and cytoadherent phenotypes of infected erythrocytes. Cell 1995, 82:101-10

6. Gardner MJ, Hall N, Fung E, White O, Berriman M, Hyman RW, Carlton JM, Pain A, Nelson KE, Bowman S, Paulsen IT, James K, Eisen JA, Rutherford K, Salzberg SL, Craig A, Kyes S, Chan MS, Nene V, Shallom S], Suh B, Peterson J, Angiuoli S, Pertea M. Allen J. Selengut J, Haft D, Mather MW, Vaidya AB, Martin DM, Fairlamb AH, Fraunholz MJ, Roos DS, Ralph SA, McFadden GI, Cummings LM, Subramanian GM, Mungall C, Venter IC, Carucci DJ, Hoffman SL, Newbold C, Davis RW, Fraser CM, Barrell B: Genome sequence of the human malaria parasite Plasmodium falciparum. Nature 2002, 4 I 9:498-5 I I.

7. Dzikowski R, Frank M, Deitsch K: Mutually Exclusive Expression of Virulence Genes by Malaria Parasites Is Regulated Independently of Antigen Production. PLoS Pathog 2006, 2:e22

8. Voss TS, Healer J, Marty AJ, Duffy MF, Thompson JK, Beeson JG, Reeder JC, Crabb BS, Cowman AF: A var gene promoter controls allelic exclusion of virulence genes in Plasmodium falciparum malaria. Nature 2006, 439(7079): 1004-8.

9. Bull PC, Marsh K: The role of antibodies to Plasmodium falciparum-infected-erythrocyte surface antigens in naturally acquired immunity to malaria. Trends Microbiol 2002, 10:55-8.

10. Smith JD, Subramanian G, Gamain B, Baruch DI, Miller LH: Classification of adhesive domains in the Plasmodium falciparum erythrocyte membrane protein I family. Mol Biochem Parasitol 2000 , I I 0:293-310.

II. Kraemer SM, Smith JD: A family affair: var genes, PfEMPI binding, and malaria disease. Curr Opin Microbiol 2006, 9:374-380.

12. Kraemer SM, Smith JD: Evidence for the importance of genetic structuring to the structural and functional specialization of the Plasmodium falciparum var gene family. Mol Microbiol 2003, 50: $1527-38$.

13. Lavstsen T, Salanti A, Jensen AT, Arnot DE, Theander TG: Subgrouping of Plasmodium falciparum 3D7 var genes based on sequence analysis of coding and non-coding regions. Malar 2003, 2:27

14. Fried $M$, Duffy PE: Two DBLgamma subtypes are commonly expressed by placental isolates of Plasmodium falciparum. Mol Biochem Parasitol 2002, I 22:201-10.

15. Rowe JA, Kyes SA, Rogerson SJ, Babiker HA, Raza A: Identification of a conserved Plasmodium falciparum var gene implicated in malaria in pregnancy. J Infect Dis 2002, I 85: I207-II.

16. Salanti A, Jensen AT, Zornig HD, Staalsoe T, Joergensen L, Nielsen MA, Khattab A, Arnot DE, Klinkert MQ, Hviid L, Theander TG: A subfamily of common and highly conserved Plasmodium falciparum var genes. Mol Biochem Parasitol 2002, I 22: I I I-5.

17. Salanti A, Staalsoe T, Lavstsen T, Jensen AT, Sowa MP, Arnot DE, Hviid $L$, Theander TG: Selective upregulation of a single distinctly structured var gene in chondroitin sulphate A-adhering Plasmodium falciparum involved in pregnancy-associated malaria. Mol Microbiol 2003, 49: 179-91.

18. Winter G, Chen Q. Flick K, Kremsner P, Fernandez V, Wahlgren M: The 3D7var5.2 (varCOMMON) type var gene family is commonly expressed in non-placental Plasmodium falciparum malaria. Molecular and Biochemical Parasitology 2003, I 27:I79-191.

19. Trimnell AR, Kraemer SM, Mukherjee S, Phippard DJ, Janes JH, Flamoe E, Su XZ, Awadalla P, Smith JD: Global genetic diversity and evolution of var genes associated with placental and severe childhood malaria. Mol Biochem Parasitol 2006, I 48: I69-I80.

20. Salanti A, Dahlback M, Turner L, Nielsen MA, Barfod L, Magistrado P, Jensen AT, Lavstsen T, Ofori MF, Marsh K, Hviid L, Theander TG: Evidence for the involvement of VAR2CSA in pregnancy-associated malaria. J Exp Med 2004, 200: I 197-203.
21. Smith JD, Deitsch KW: Pregnancy-associated malaria and the prospects for syndrome-specific antimalaria vaccines. J Exp Med 2004, 200:1093-7.

22. Freitas-Junior LH, Bottius E, Pirrit LA, Deitsch KW, Scheidig C, Guinet F, Nehrbass U, Wellems TE, Scherf A: Frequent ectopic recombination of virulence factor genes in telomeric chromosome clusters of $\boldsymbol{P}$. falciparum. Nature 2000, 407:1018-22.

23. Taylor HM, Kyes SA, Newbold $\mathrm{Cl}$ : Var gene diversity in Plasmodium falciparum is generated by frequent recombination events. Mol Biochem Parasitol 2000, I 1 0:391-7.

24. Ward CP, Clottey GT, Dorris M, Ji DD, Arnot DE: Analysis of Plasmodium falciparum PfEMP-I/var genes suggests that recombination rearranges constrained sequences. Mol Biochem Parasitol 1999, 102:167-77.

25. Robinson BA, Welch TL, Smith JD: Widespread functional specialization of Plasmodium falciparum erythrocyte membrane protein I family members to bind CD36 analysed across a parasite genome. Mol Microbiol 2003, 47: I 265-78.

26. Biggs BA, Anders RF, Dillon HE, Davern KM, Martin M, Petersen C, Brown GV: Adherence of infected erythrocytes to venular endothelium selects for antigenic variants of Plasmodium falciparum. J Immunol 1992, 149:2047-2054.

27. Bourke PF, Holt DC, Sutherland CJ, Kemp DJ: Disruption of a novel open reading frame of Plasmodium falciparum chromosome 9 by subtelomeric and internal deletions can lead to loss or maintenance of cytoadherence. Mol Biochem Parasitol 1996, 82:25-36.

28. Day KP, Karamalis F, Thompson J, Barnes DA, Peterson C, Brown H, Brown GV, Kemp DJ: Genes necessary for expression of a virulence determinant and for transmission of Plasmodium falciparum are located on a 0.3-megabase region of chromosome 9. Proc Natl Acad Sci U S A 1993, 90:8292-8296.

29. Roberts DJ, Craig AG, Berendt AR, Pinches R, Nash G, Marsh K, Newbold $\mathrm{Cl}$ : Rapid switching to multiple antigenic and adhesive phenotypes in malaria. Nature 1992, 357:689-92.

30. 2007 [http://www.broad.mit.edu]

31. Johnson JK, Swerlick RA, Grady KK, Millet P, Wick TM: Cytoadherence of Plasmodium falciparum-infected erythrocytes to microvascular endothelium is regulatable by cytokines and phorbol ester. J Infect Dis 1993, 167:698-703.

32. Xiao L, Yang C, Dorovini-Zis K, Tandon NN, Ades EW, Lal AA Udhayakumar V: Plasmodium falciparum: involvement of additional receptors in the cytoadherence of infected erythrocytes to microvascular endothelial cells. Exp Parasitol 1996, 84:42-55.

33. Udeinya IJ, Graves PM, Carter R, Aikawa M, Miller LH: Plasmodium falciparum: effect of time in continuous culture on binding to human endothelial cells and amelanotic melanoma cells. Exp Parasitol 1983, 56:207-14.

34. Robson $\mathrm{KJH}$, Walliker D, Creasey A, McBride J, Beale G, Wilson RJM: Cross-contamination of Plasmodium cultures. Parasitology Today 1992, 8:38-39.

35. Bhasin VK, Trager W: Gametocyte-forming and non-gametocyte-forming clones of Plasmodium falciparum. Am J Trop Med Hyg 1984, 33:534-537.

36. Ponnudurai T, Leeuwenberg AD, Meuwissen JH: Chloroquine sensitivity of isolates of Plasmodium falciparum adapted to in vitro culture. Trop Geogr Med 198I, 33:50-54.

37. Mu J, Awadalla P, Duan J, McGee KM, Joy DA, McVean GA, Su XZ: Recombination hotspots and population structure in Plasmodium falciparum. PLoS Biol 2005, 3:e335.

38. Peterson DS, Miller LH, Wellems TE: Isolation of multiple sequences from the Plasmodium falciparum genome that encode conserved domains homologous to those in erythrocyte- binding proteins. Proc Natl Acad Sci U S A 1995, 92:7100-4.

39. Kyes S, Taylor H, Craig A, Marsh K, Newbold C: Genomic representation of var gene sequences in Plasmodium falciparum field isolates from different geographic regions. Mol Biochem Parasitol 1997, 87:235-8.

40. Fowler EV, Peters JM, Gatton ML, Chen N, Cheng Q: Genetic diversity of the DBLalpha region in Plasmodium falciparum var genes among Asia-Pacific isolates. Mol Biochem Parasitol 2002. 120:1 17-26.

4I. Wunderlich G, Alves FP, Golnitz U, Tada MS, Camargo EF, Pereira-daSilva LH: Rapid turnover of Plasmodium falciparum var gene transcripts and genotypes during natural non-symptomatic infections. Rev Inst Med Trop Sao Paulo 2005, 47: I95-20।

42. Fernandez $V$, Chen Q, Sundstrom A, Scherf A, Hagblom P, Wahlgren $M$ : Mosaic-like transcription of var genes in single Plasmodium falciparum parasites. Mol Biochem Parasitol 2002, I 2 I:195-203. 
43. Kirchgatter K, Mosbach R, del Portillo HA: Plasmodium falciparum: DBL-I var sequence analysis in field isolates from central Brazil. Exp Parasitol 2000, 95: 154-7.

44. Afonso Nogueira P, Wunderlich G, Shugiro Tada M, d'Arc Neves Costa J, Jose Menezes M, Scherf A, Pereira-da-Silva LH: Plasmodium falciparum: analysis of transcribed var gene sequences in natural isolates from the Brazilian Amazon region. Exp Parasitol 2002, I I I: I II-20.

45. Kaestli M, Cortes A, Lagog M, Ott M, Beck HP: Longitudinal assessment of Plasmodium falciparum var gene transcription in naturally infected asymptomatic children in Papua New Guinea. J Infect Dis 2004, I 89: 1942-I95।.

46. Bull PC, Berriman M, Kyes S, Quail MA, Hall N, Kortok MM, Marsh K Newbold Cl: Plasmodium falciparum Variant Surface Antigen Expression Patterns during Malaria. PLoS Pathogens 2005, I:e26.

47. Taylor HM, Kyes SA, Harris D, Kriek N, Newbold Cl: A study of var gene transcription in vitro using universal var gene primers. Mol Biochem Parasitol 2000, 1 05: $13-23$.

48. Kirchgatter K, Portillo Hdel A: Association of severe noncerebral Plasmodium falciparum malaria in Brazil with expressed PfEMPI DBLI alpha sequences lacking cysteine residues. Mol Med 2002, 8: 16-23.

49. Kraemer SM, Gupta L, Smith JD: New tools to identify var sequence tags and clone full-length genes using type-specific primers to Duffy binding-like domains. Mol Biochem Parasitol 2003, I 29:91-102.

50. Duffy MF, Brown GV, Basuki W, Krejany EO, Noviyanti R, Cowman AF, Reeder JC: Transcription of multiple var genes by individual, trophozoite-stage Plasmodium falciparum cells expressing a chondroitin sulphate $\mathbf{A}$ binding phenotype. Mol Microbiol 2002, 43: $1285-93$

5I. Rowe JA, Moulds JM, Newbold $\mathrm{Cl}$, Miller $\mathrm{LH}$ : $\boldsymbol{P}$. falciparum rosetting mediated by a parasite-variant erythrocyte membrane protein and complement-receptor I. Nature 1997, 388:292-5.

52. Wellcome Trust Sanger Institute [http://www.sanger.ac.uk Projects/P_falciparum]

53. Thompson JK, Rubio JP, Caruana S, Brockman A, Wickham ME, Cowman AF: The chromosomal organization of the Plasmodium falciparum var gene family is conserved. Mol Biochem Parasitol 1997. 87:49-60.

54. Kyes SA, Christodoulou Z, Raza A, Horrocks P, Pinches R, Rowe JA, Newbold $\mathrm{Cl}$ : A well-conserved Plasmodium falciparum var gene shows an unusual stage-specific transcript pattern. Mol Micro biol 2003, 48: $1339-1348$.

55. Voss TS, Thompson JK, Waterkeyn J, Felger I, Weiss N, Cowman AF, Beck HP: Genomic distribution and functional characterisation of two distinct and conserved Plasmodium falciparum var gene '5' flanking sequences. Mol Biochem Parasitol 2000 , 107:103-15.

56. Carver TJ, Rutherford KM, Berriman M, Rajandream MA, Barrell BG, Parkhill J: ACT: the Artemis Comparison Tool. Bioinformatics 2005, 2 1:3422-3423

57. Singh SK, Hora R, Belrhali H, Chitnis CE, Sharma A: Structural basis for Duffy recognition by the malaria parasite Duffy-bindinglike domain. Nature 2006, 439:74I-744.

58. Tolia NH, Enemark EJ, Sim BK, Joshua-Tor L: Structural basis for the EBA-I75 erythrocyte invasion pathway of the malaria parasite Plasmodium falciparum. Cell 2005, I22:183-193.

59. Frank M, Deitsch K: Activation, silencing and mutually exclusive expression within the var gene family of Plasmodium falciparum. Int | Parasitol 2006, 36:975-985.

60. Duraisingh MT, Voss TS, Marty AJ, Duffy MF, Good RT, Thompson JK, Freitas-Junior LH, Scherf A, Crabb BS, Cowman AF: Heterochromatin Silencing and Locus Repositioning Linked to Regulation of Virulence Genes in Plasmodium falciparum. Cell 2005, | 2 I: | 3-24.

6I. Freitas-Junior LH, Hernandez-Rivas R, Ralph SA, Montiel-Condado D, Ruvalcaba-Salazar OK, Rojas-Meza AP, Mancio-Silva L, Leal-Silvestre RJ, Gontijo AM, Shorte S, Scherf A: Telomeric Heterochromatin Propagation and Histone Acetylation Control Mutually Exclusive Expression of Antigenic Variation Genes in Malaria Parasites. Cell 2005, 1 21:25-36.

62. Verger A, Crossley M: Chromatin modifiers in transcription and DNA repair. Cellular and Molecular Life Sciences (CMLS) 2004, 61:2154-2162.

63. Lombard DB, Chua KF, Mostoslavsky R, Franco S, Gostissa M, Alt FW: DNA Repair, Genome Stability, and Aging. Cell 2005, I 20:497-5 I 2.

64. Jensen AT, Magistrado P, Sharp S, Joergensen L, Lavstsen T, Chiucchiuini A, Salanti A, Vestergaard LS, Lusingu JP, Hermsen R, Sauerwein R, Christensen J, Nielsen MA, Hviid L, Sutherland C, Staalsoe T, Theander TG: Plasmodium falciparum associated with severe child- hood malaria preferentially expresses PfEMPI encoded by group A var genes. J Exp Med 2004, 199: I 179-90.

65. Gupta S, Maiden MC, Feavers IM, Nee S, May RM, Anderson RM: The maintenance of strain structure in populations of recombining infectious agents. Nat Med 1996, 2:437-442.

66. Bull PC, Kortok M, Kai O, Ndungu F, Ross A, Lowe BS, Newbold Cl, Marsh K: Plasmodium falciparum-infected erythrocytes: agglutination by diverse Kenyan plasma is associated with severe disease and young host age. I Infect Dis 2000, I 82:252-9.

67. Bull PC, Lowe BS, Kortok M, Marsh K: Antibody recognition of Plasmodium falciparum erythrocyte surface antigens in Kenya: evidence for rare and prevalent variants. Infect Immun 1999, 67:733-9.

68. Kyes S, Horrocks P, Newbold C: Antigenic variation at the infected red cell surface in malaria. Annu Rev Microbiol 200I, 55:673-707

69. Chen Q, Heddini A, Barragan A, Fernandez V, Pearce SF, Wahlgren M: The semiconserved head structure of Plasmodium falciparum erythrocyte membrane protein I mediates binding to multiple independent host receptors. I Exp Med 2000, 192:1-10.

70. Horrocks P, Pinches R, Christodoulou Z, Kyes SA, Newbold Cl: Variable var transition rates underlie antigenic variation in malaria. Proc Natl Acad Sci U S A 2004, I 0 I : I I I29-34.

7I. Gordon D, Abaiian C, Green P: Consed: a graphical tool for sequence finishing. Genome Res 1998, 8:195-202.

72. Hinterberg K, Scherf A: PFGE: improved conditions for rapid and high-resolution separation of Plasmodium falciparum chromosomes. Parasitol Today 1994, 10:225.

73. Kyes S, Pinches R, Newbold C: A simple RNA analysis method shows var and rif multigene family expression patterns in Plasmodium falciparum. Mol Biochem Parasitol 2000, I 05:3 | I-5.

74. Hall N, Pain A, Berriman M, Churcher C, Harris B, Harris D, Mungal K, Bowman S, Atkin R, Baker S, Barron A, Brooks K, Buckee CO, Burrows C, Cherevach I, Chillingworth C, Chillingworth T, Christodoulou Z, Clark L, Clark R, Corton C, Cronin A, Davies R, Davis P, Dear P Dearden F, Doggett J, Feltwell T, Goble A, Goodhead I, Gwilliam R, Hamlin N, Hance Z, Harper D, Hauser H, Hornsby T, Holroyd S, Horrocks $P$, Humphray $S$, Jagels $K$, James $K D$, Johnson $D$, Kerhornou $A$, Knights A, Konfortov B, Kyes S, Larke N, Lawson D, Lennard N, Line A, Maddison M, McLean J, Mooney P, Moule S, Murphy L, Oliver K, Ormond D, Price C, Quail MA, Rabbinowitsch E, Rajandream MA, Rutter S, Rutherford KM, Sanders M, Simmonds M, Seeger K, Sharp S, Smith R, Squares R, Squares S, Stevens K, Taylor K, Tivey A, Unwin L, Whitehead S, Woodward J, Sulston JE, Craig A, Newbold C, Barrell BG: Sequence of Plasmodium falciparum chromosomes I, 3-9 and I3. Nature 2002, 4I9:527-3I.

75. Kurtz S, Phillippy A, Delcher A, Smoot M, Shumway M, Antonescu C, Salzberg S: Versatile and open software for comparing large genomes. Genome Biology 2004, 5:R I2

76. Swofford DL, Olson GJ, Waddell PJ, Hillis DM: Phylogenetic Inference. In Molecular Systematics Edited by: Hillis DM, Moritz C and Mable BK. Sinauer Associates; 1996:407-5 I4.

77. Rutherford K, Parkhill J, Crook J, Horsnell T, Rice P, Rajandream MA Barrell B: Artemis: sequence visualization and annotation. Bioinformatics 2000, 16:944-945

78. Viebig NK, Gamain B, Scheidig C, Lepolard C, Przyborski J, Lanzer M, Gysin J, Scherf A: A single member of the Plasmodium falciparum var multigene family determines cytoadhesion to the placental receptor chondroitin sulphate A. EMBO Rep 2005, 6:775-78|

Publish with Bio Med Central and every scientist can read your work free of charge

"BioMed Central will be the most significant development for disseminating the results of biomedical research in our lifetime. "

Sir Paul Nurse, Cancer Research UK

Your research papers will be:

- available free of charge to the entire biomedical community

- peer reviewed and published immediately upon acceptance

- cited in PubMed and archived on PubMed Central

- yours - you keep the copyright
BioMedcentral 